\title{
Ground magnetic perturbations associated with the standing toroidal mode oscillations in the magnetosphere-ionosphere system
}

\author{
Hiroyuki Nakata $^{1 *}$, Shigeru Fujita ${ }^{1,2}$, Akimasa Yoshikawa ${ }^{3}$, Masahiro Itonaga $^{4}$, and Kiyohumi Yumoto ${ }^{3}$ \\ ${ }^{1}$ Department of Geophysics, Kyoto University, Kyoto 606-8502, Japan \\ ${ }^{2}$ Meteorological College, Kashiwa 277-0852, Japan \\ ${ }^{3}$ Department of Earth and Planetary Sciences, Kyushu University, Fukuoka 812-8581, Japan \\ ${ }^{4}$ Faculty of Education, Yamaguchi University, Yamaguchi 753-8513, Japan
}

(Received October 15, 1999; Revised July 10, 2000; Accepted August 17, 2000)

\begin{abstract}
The behavior of toroidal mode oscillations of standing Alfvén waves (refer to as standing Alfvén oscillations) in the coupled magnetosphere-ionosphere system is investigated using a trapezoid-shape magnetosphere model. It is found that the magnetic perturbation is transmitted across the ionosphere differently in the two cases where the ionospheric electric field perturbation is static (Pedersen conductivity $>$ Hall conductivity) and where it is inductive (Pedersen conductivity $<$ Hall conductivity). It is noted that the ionospheric Hall current for the inductive condition shields the magnetic field perturbation. The north-south asymmetry of the conjugate ground magnetic perturbations is calculated by using a trapezoid model with the ionospheric and magnetospheric parameters based on the IGRF and IRI. It is revealed that the ionospheric electric field is almost static for the fundamental mode oscillation, whereas inductive for the higher harmonic ones. It is also found that the north-south asymmetry of the ground magnetic perturbations depends not only on the $L$-value but also on magnetic longitude; this is because the ionosphere and magnetic field conditions are not uniform as a function of longitude.
\end{abstract}

\section{Introduction}

Magnetohydrodynamic (MHD) waves excited in the magnetosphere cause the ultra-low-frequency (ULF) pulsations observed on the ground. The extensive ground network observations carried out in the STEP period revealed many important features of ULF pulsations. For example, Yumoto et al. (1994) showed that, from the $210^{\circ}$ magnetic meridian ground network observations, most Pc 3-4 pulsations associated with the interplanetary impulses are standing Alfvén oscillations of the magnetic field lines. It is widely accepted that standing Alfvén oscillations are excited by a field line resonance (FLR) (Chen and Hasegawa, 1974; Southwood, 1974). Tamao (1964) first presented the idea of FLR. Since there are many observational findings based on ground magnetic observations, we need to evaluate theoretically the ground magnetic perturbations associated with standing Alfvén oscillations. To evaluate them, we need to take ionospheric modification of standing Alfvén oscillations into consideration.

The ionosphere has two important effects on the behavior of standing Alfvén oscillations. One is ionospheric Joule dissipation which causes damping of standing Alfvén oscillations (Newton et al., 1978; Allan and Knox, 1979a). Allan and Knox (1979a) analytically derived the damping rate due

\footnotetext{
* Now at Solar-Terrestrial Environment Laboratory, Nagoya University, 3-13, Honohara, Toyokawa, 442-8507, Japan.

Copy right (C) The Society of Geomagnetism and Earth, Planetary and Space Sciences (SGEPSS); The Seismological Society of Japan; The Volcanological Society of Japan; The Geodetic Society of Japan; The Japanese Society for Planetary Sciences.
}

to the ionospheric Pedersen current. They revealed that the damping rate is maximized when the height-integrated Pedersen conductivity $\left(\Sigma_{P}\right)$ is equal to the Alfvén conductance $\left(\Sigma_{A}\right)$ of the resonant field line. Furthermore, for $\Sigma_{P}>\Sigma_{A}$, the damping rate is proportional to $\Sigma_{P}$, and for $\Sigma_{P}<\Sigma_{A}$, the damping rate is inversely proportional to $\Sigma_{P}$. Note that the Hall conductivity was excluded in their analysis.

Another important effect of the ionosphere results from the ionospheric Hall current, which causes a conversion from Alfvén wave to fast-mode wave (e.g., Tamao, 1965) and produces the ground magnetic perturbation (e.g., Nishida, 1964, 1978; Inoue and Horowitz, 1966a,b; Hughes and Southwood, 1976a,b). Tamao (1984) argued that the Alfvén and the fast mode waves have static and inductive electric fields, respectively. That is, the electric field of the Alfvén wave is given by

$$
\delta \mathbf{E}_{\perp, s t a}=-\nabla_{\perp} \Phi,
$$

and that of the fast mode wave is given by

$$
\delta \mathbf{E}_{\perp, \text { ind }}=-\frac{\partial}{\partial t} \nabla_{\perp} \times(\hat{e} \Psi),
$$

where $\Phi$ and $\Psi$ are scalar functions and $\hat{e}$ is a unit vector directed parallel to the main magnetic field. These equations show that the Alfvén wave and the fast mode wave have divergent and rotational horizontal electric fields, respectively. These two electric fields are connected to one another via the Hall current. As regards the ground magnetic perturbation, Poulter and Allan (1985) and Allan (1995) investigated the ground magnetic perturbation associated with standing Alfvén oscillations. They calculated the ground magnetic 


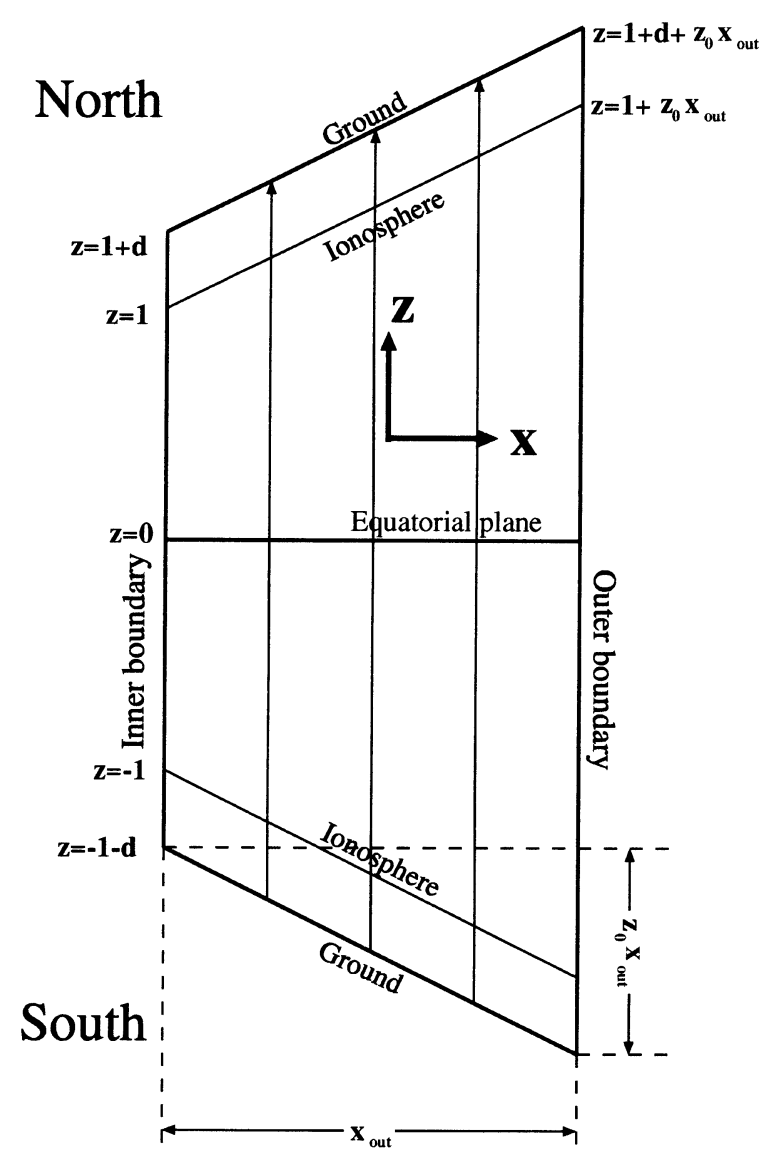

Fig. 1. The schematic figure of the model used in the present study. The inner and outer boundaries are located at $x=0$ and $x_{0}$. The ionospheres at $x=0$ are located at $z= \pm 1$ ( \pm refer to the northern and southern ionospheres, respectively) and at $x=x_{\text {out }}, z= \pm\left(1+z_{0} x_{\text {out }}\right)$. The distance between the ionosphere and the Earth is $d$. The $z$ coordinate of the magnetic equatorial plane is $z=0$.

perturbations using the following procedures; first, the electric field of the Alfvén wave in the ionosphere with only $\Sigma_{P}$ is obtained; next, the Hall current is derived as the product of the ionospheric electric field thus obtained and the height-integrated Hall conductivity $\left(\Sigma_{H}\right)$. This Hall current produces the ground magnetic perturbation. The procedure is not self-consistent because only $\Sigma_{P}$ is taken into account in the first step.

Recently, Yoshikawa and Itonaga (1996) and Buchert and Budnik (1997) examined the self-consistent procedure. They reported that the ionospheric Hall current modifies the behavior of standing Alfvén oscillations when the wave frequency becomes higher or $\Sigma_{H} / \Sigma_{P}$ becomes larger. That is, the damping of standing Alfvén oscillations is controlled not only by the Pedersen conductivity but also by the Hall conductivity for this case. This fact also indicates that, in order to obtain theoretically the ground magnetic perturbation associated with standing Alfvén oscillations, we need to consider both the Pedersen conductivity and the Hall conductivity in the ionospheric boundary condition. In the present paper, to evaluate the ground magnetic perturbation under the anisotropically conducting ionosphere, we calculate selfconsistently electromagnetic perturbations associated with standing Alfvén oscillations in the coupled magnetosphere- ionosphere system.

The ionosphere and the magnetosphere are usually regarded as asymmetric along a magnetic field line with respect to the equator. Then, it is likely that this asymmetry of the magnetosphere-ionosphere system yields the asymmetry between the conjugate ground magnetic perturbations. Therefore, it is another target of the present paper to evaluate the north-south asymmetry of the ground magnetic perturbations based on model calculations.

The structure of the present paper is as follows. In Section 2, we explain the model and the basic equations. In Section 3, using the numerical results, we show how the behavior of standing Alfvén oscillations is controlled by the magnetospheric and ionospheric parameters. In the latter part of this section, the north-south asymmetry of the ground magnetic perturbations due to the asymmetric parameters investigated. In Section 4, the conjugate asymmetry of the ground magnetic perturbations is calculated using a trapezoid model with the ionospheric and magnetospheric parameters obtained from the IRI and IGRF. Then a comparison of results of the present study and the observational study using data from the $210^{\circ} \mathrm{MM}$ chain is carried out. In the last section, the effect of the additional parameters are discussed and the results in the present paper are summarized.

\section{Model and Basic Equations \\ 2.1 Model}

The model is schematically shown in Fig. 1. A trapezoidshape magnetosphere model is used in the present study. Even though standing Alfvén oscillations is ideally a 1D phenomenon, the latitudinal width of standing Alfvén oscillations must be considered because the pure standing Alfvén oscillation without latitudinal spread (e.g., Poulter and Allan, 1985) does not produce a ground magnetic perturbation. Physically speaking, the ionospheric Hall current makes the Alfvén mode electromagnetic perturbation spread horizontally in the ionosphere.

The model includes the magnetosphere and an infinitely thin ionosphere, which has height-integrated Pedersen and Hall conductivities. The main magnetic field is assumed to be straight and inclined to the ionosphere in the trapezoid model. A free parameter $z_{0}$ assigns the magnetic inclination to the ionosphere. The spatially non-uniform Alfvén speed $\left(V_{A}\right)$ is given by the following equation:

$$
V_{A}(x, z)=\left\{V_{A, i s}-V_{A, e q}(x)\right\}\left\{\frac{z}{z_{i s}(x)}\right\}^{2}+V_{A, e q}(x),
$$

where $V_{A, i s}$ and $V_{A, e q}(x)$ are respectively the Alfvén speed in the ionosphere and in the equatorial plane. $z$ axis is parallel to the main magnetic field and $z$ coordinates of the ionosphere is denoted by $z_{i s}$ which is a function of $x$. $V_{A, i s}$ is considered to be constant in each ionosphere. $V_{A, e q}$ monotonically varies with $x$ like

$$
V_{A, e q}(x)=\left(V_{A, e q}^{o u t}-V_{A, e q}^{i n}\right) \frac{x}{x_{\text {out }}}+V_{A, e q}^{\text {in }} .
$$

Here $V_{A, e q}^{\text {out }}$ and $V_{A, e q}^{\text {in }}$ are the Alfvén speed in the equatorial plane at the outer boundary $\left(x=x_{\text {out }}\right)$ and that in the equatorial plane at the inner boundary $\left(x=x_{i n}=0\right)$. In the 
present paper, $V_{A, e q}^{\text {in }} / V_{A, e q}^{\text {out }}=4, x_{\text {out }}=0.25, V_{A, i s}=10$ and the height of the ionosphere $(d)=0.01$ are chosen unless we specify these values otherwise. The numbers of the grid points are 12 in the $x$ direction and 31 in the $z$ direction. $V_{A}$ is normalized to $V_{A, e q}\left(x_{0}\right)$, where $x_{0}=6 x_{\text {out }} / 11$. We analyze standing Alfvén oscillations on the field line at $x=x_{0}$.

\subsection{Basic equation and ionospheric boundary condi-} tions

In the cold MHD regime, electromagnetic field perturbations are given by

$$
\begin{aligned}
& \nabla \times \delta \boldsymbol{E}_{\perp}=-\frac{\partial \delta \boldsymbol{B}}{\partial t}, \\
& \nabla_{\perp} \times \delta \boldsymbol{B}=\frac{1}{V_{A}^{2}(x, z)} \frac{\partial \delta \boldsymbol{E}_{\perp}}{\partial t}
\end{aligned}
$$

(e.g., Lee and Lysak, 1989), where $\delta \boldsymbol{E}_{\perp}$ and $\delta \boldsymbol{B}$ are the electric field perturbation perpendicular to the main magnetic field and the magnetic field perturbation, respectively. Eliminating $\delta \boldsymbol{B}$ from Eqs. (5) and (6), we have the electric field perturbation at frequency $\omega$ is given by

$$
\frac{\omega^{2}}{V_{A}^{2}(x, z)} \delta \boldsymbol{E}_{\perp}=\nabla_{\perp} \times\left(\nabla \times \delta \boldsymbol{E}_{\perp}\right) .
$$

The orthogonal grid system necessary for the finite difference scheme is obtained through the boundary-fitted coordinate technique; the original coordinates, $(x, z)$, are transformed to the boundary-fitted coordinates, $(\xi, \eta)$, using following relations:

$$
\begin{aligned}
& x=\frac{1+\xi}{2} x_{\text {out }}, \\
& z=\frac{\eta}{2}\left(2+(1+\xi) x_{\text {out }} z_{0}\right) .
\end{aligned}
$$

In the $(\xi, \eta)$ system, the trapezoid model has a square shape defined by $|\xi| \leq 1$ and $|\eta| \leq 1$.

Tamao $(1984,1986)$ has presented the set of equations describing the ionosphere boundary conditions for oblique magnetic field. We need alternative expressions for Tamao's equations to permit further numerical analysis. Using mathematical manipulation similar to that employed by Itonaga et al. (1995), we obtain

$$
\begin{aligned}
& i \omega \mu_{0} \Sigma_{P} \nabla \delta \boldsymbol{E}_{t}+i \omega \mu_{0} \Sigma_{H} \hat{N}\left(\nabla \times \delta \boldsymbol{E}_{t}\right) \\
& =-(\hat{N} \cdot \hat{z})(\hat{z} \cdot \nabla)\left(\nabla \delta \boldsymbol{E}_{\perp}\right)-\left(\frac{\omega}{V_{A}}\right)^{2}\left(\hat{N}_{\perp} \delta \boldsymbol{E}_{\perp}\right) \\
& i \omega \mu_{0} \Sigma_{P} \hat{N}\left(\nabla \times \delta \boldsymbol{E}_{t}\right)-i \omega \mu_{0} \Sigma_{H} \nabla \delta \boldsymbol{E}_{t} \\
& \quad=-\hat{N}\left(\nabla \times \delta \boldsymbol{E}_{\perp}\right)(\nabla \hat{N})-\hat{N}(\hat{N} \cdot \nabla)\left(\nabla \times \delta \boldsymbol{E}_{\perp}\right) \\
& \quad+\frac{\hat{N}\left(\nabla \times \delta \boldsymbol{E}_{\perp}\right)}{d},
\end{aligned}
$$

where $\hat{z}$ is the unit vector along $z$ axis or the main magnetic field. Here $\delta \boldsymbol{E}_{t}$ is the wave electric field tangential to the ionosphere and $\hat{N}$ is the unit vector normal to the ionosphere. The ratio of the second term on the r.h.s. of Eq. (10) to the first term is estimated as $\left(\omega / V_{A}\right)^{2}\left(\hat{N}_{\perp} \delta \boldsymbol{E}_{\perp}\right) /(\hat{N} \cdot \hat{z})(\hat{z} \cdot \nabla)\left(\nabla \delta \boldsymbol{E}_{\perp}\right) \simeq$ $\left(l_{\perp} / l_{\|}\right) \cot I$ where $l_{\|}$and $l_{\perp}$ are the field-aligned and latitudinal scales of standing Alfvén oscillations and $I$ is the inclination angle. Bearing in mind that $l_{\perp} \ll l_{\|}$, this ratio is much smaller than 1 unless $I \simeq 0$ (in the equatorial ionosphere). Therefore, the second term is neglected in the present study. In addition, the first term in r.h.s. of Eq. (11) vanishes in the present model when $\hat{N}$ is uniform.

The magnetic field perturbation just below the ionosphere corresponds to that on the ground for the present approximation in which the latitudinal width of the wave $\gg d$. We consider the case where the azimuthal wave number ( $m$ number) is 0 , which means that there is no coupling of standing Alfvén oscillations and the fast-mode oscillation in the magnetosphere.

\section{Numerical Results}

In this section, the ground magnetic perturbation associated with standing Alfvén oscillations is investigated. Before showing the results, we shall verify that the model derives physically correct results. This is done by the comparing the numerical values of the ionospheric electric field with the previous works of Yoshikawa and Itonaga (1996) and Yoshikawa et al. (1999) as follows.

\subsection{Verification of our model}

The behavior of standing Alfvén oscillations in the trapezoid model is controlled by the three magnetosphere/ionosphere parameters, i.e. the ionospheric conductivity, the inclination of magnetic field lines and the ratio of the Alfvén speed in the ionosphere to that in the equatorial plane. Note that the trapezoid model has straight field lines unlike the
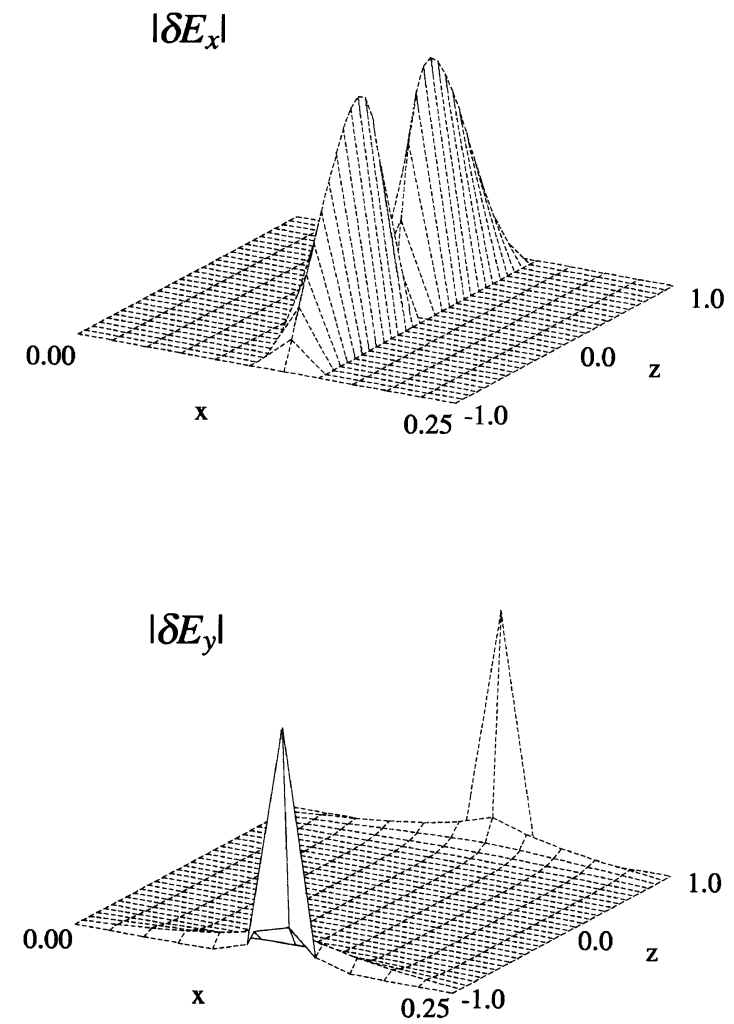

Fig. 2. The 2D profiles of the electric field intensity associated with the standing Alfvén oscillation $\left(\left|\delta E_{x}\right|\right)$ and that of the fast mode wave induced through the ionospheric Hall current $\left(\left|\delta E_{y}\right|\right)$. Parameters are $\bar{\Sigma}_{P 0}=\bar{\Sigma}_{P 0}=100, z_{0}=0$ and $V_{A, i s}=10$. Note that the main magnetic field is perpendicular to the ionosphere $\left(z_{0}=0\right)$. 

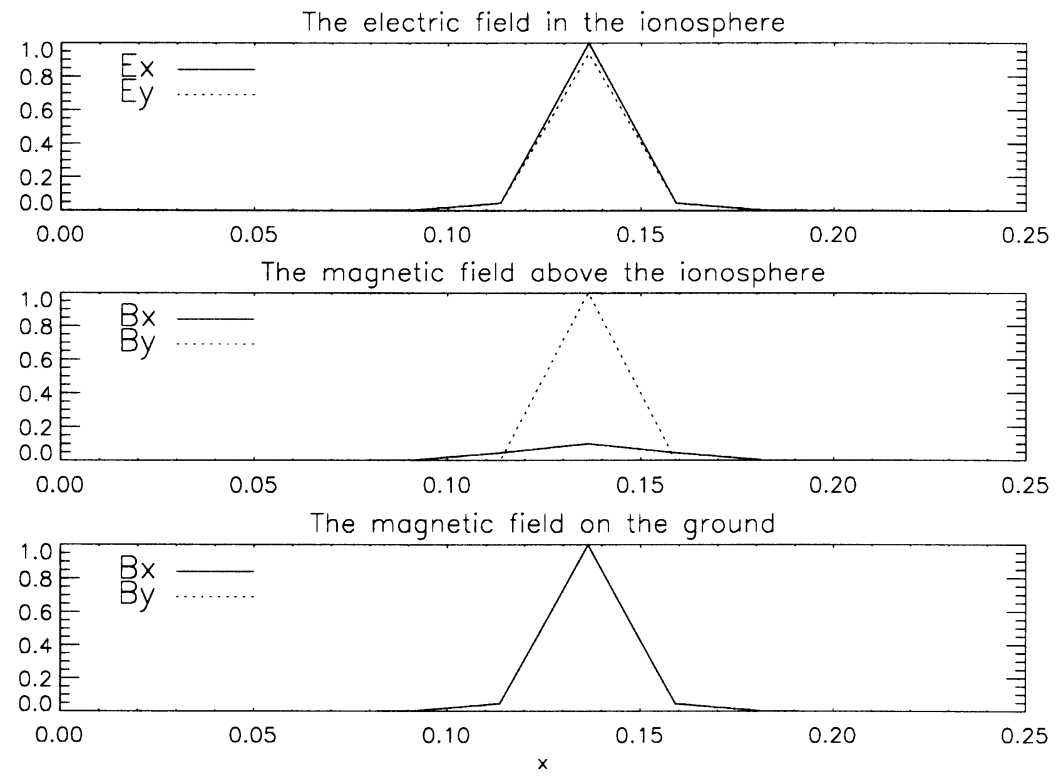

Fig. 3. Latitudinal ( $x$-direction) intensity profiles of the electric field perturbations in the ionosphere (top), those of the magnetic field perturbations just above the ionosphere (middle) and those on the ground (bottom). The parameters are same as that used in Fig. 2. In each panel, the $x$ and $y$ components are represented with the solid and dotted lines, respectively.

curved ones in a dipole field. The dipole magnetic field features the convergence of magnetic flux toward the ionosphere leading to an increase in electromagnetic field intensity toward the ionosphere. This effect is not included in the present trapezoid model.

First, spatial structures of the second mode of standing Alfvén oscillations are shown in Fig. 2. This figure shows electric field perturbations associated with the second harmonic standing Alfvén oscillation $\left(\delta E_{x}\right)$ and the fast mode wave $\left(\delta E_{y}\right)$ generated through the ionospheric Hall current. We use $z_{0}=0, \Sigma_{P}=\Sigma_{H}=100 \Sigma_{A 0}$ in both ionospheres, where $\Sigma_{A 0}=1 / \mu_{0} V_{A, e q}\left(x_{0}\right) . \Sigma_{P}$ and $\Sigma_{H}$ are normalized as

$$
\bar{\Sigma}_{P 0}=\frac{\Sigma_{P}}{\Sigma_{A 0}}, \quad \bar{\Sigma}_{H 0}=\frac{\Sigma_{H}}{\Sigma_{A 0}},
$$

throughout the present study. Note that the Alfvén conductance of the second harmonic Alfvén oscillation normalized to $\Sigma_{A 0}$ is about 0.23 . Since $\Sigma_{P}$ is larger than $\Sigma_{A 0}$ in Fig. 2, standing Alfvén oscillations have nodes in the ionosphere. Figure 3 shows latitudinal profiles of electric field perturbations in the ionosphere (top), the magnetic field perturbations just above the ionosphere (middle) and those on the ground (bottom). The parameters used for Fig. 3 are as same as for Fig. 2. The solid and dotted lines represent the $x$ and $y$ components respectively in each panels. Figures 2 and 3 clearly demonstrate that the Alfvén-mode electric field $\left(\delta E_{x}\right)$ shows the standing-wave behavior and that the fast-mode electric field $\left(\delta E_{y}\right)$ exhibits an evanescent behavior. Note that $\delta B_{y}$ on the ground is zero. This is consistent with the theoretical analysis that $\delta B_{y}$ on the ground vanishes when the azimuthal wave number, $m=0$ (e.g., Nishida, 1978). The disappearance of $\delta B_{y}$ on the ground indicates verifies our numerical calculations.

Next, we investigate the dependence of the ionospheric electric field on the ionospheric conductivity. Yoshikawa and
Itonaga (1996) and Yoshikawa et al. (1999) discussed the importance of $\Sigma_{H}$ that yields the inductive electric field from the static one. From their results, $\Sigma_{H}$ also contributes to the reflection of the Alfvén wave. That is, the ionospheric inductive electric field produced by the Hall current reduces the resultant static field. The total ionospheric electric field including the effect of $\Sigma_{H}$ is given by equation (11) in Yoshikawa et al. (1999). We rewrite the equation as follows:

$$
\frac{\delta E_{x, i s}}{\delta E_{x}^{i}}=\frac{2}{1+\hat{\Sigma}_{P}+k_{\|}^{A} \hat{\Sigma}_{H}^{2} / D},
$$

where $\delta E_{x}^{i}$ is the incident electric field, $D=k_{F}+i k_{x}$ $\operatorname{coth}\left(k_{x} d\right)+k_{\|}^{A} \hat{\Sigma}_{P}, \hat{\Sigma}_{P}=\mu_{0} V_{A} \Sigma_{P}, \hat{\Sigma}_{H}=\mu_{0} V_{A} \Sigma_{H}, k_{\|}^{A}=$ $\omega / V_{A}, k_{F}=\sqrt{\left(k_{\|}^{A}\right)^{2}-k_{x}^{2}}$ and $k_{x}$ is the wave number in the $x$ direction.

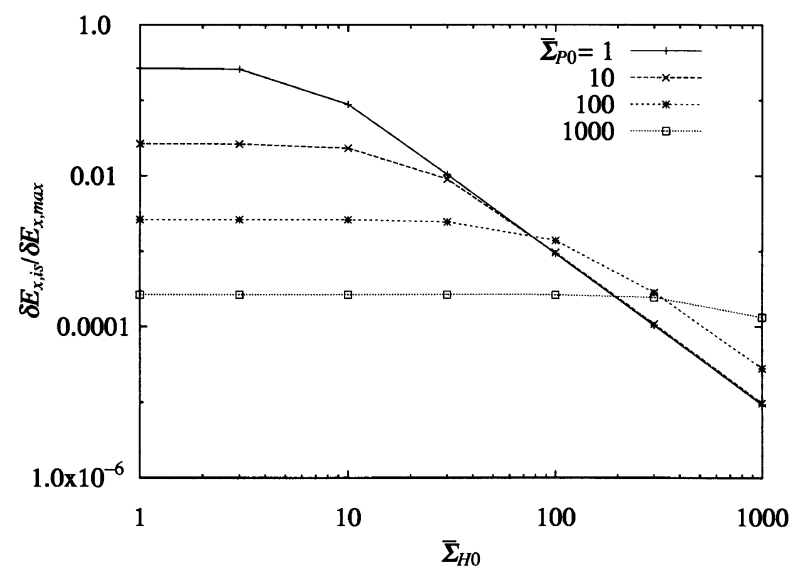

Fig. 4. $\delta E_{x, i s} / \delta E_{x, \max }$ of the second mode standing Alfvén oscillation as functions of $\bar{\Sigma}_{H 0}$. The ratios for 4 cases of $\bar{\Sigma}_{P 0}(=1,10,100$ and 1000) are shown. 
Next the numerical results are verified using Eq. (13). To do so, the electric field in the ionosphere normalized by the maximum electric field along the resonant field line of the second harmonic mode $\left(\delta E_{x, i s} / \delta E_{x, \max }\right)$ is shown as a function of $\bar{\Sigma}_{H 0}$ in Fig. 4. Equation (13) is derived under the situation of the entrance and reflection of the Alfvén wave. Although such a situation differs from the standing oscillation, Equation (13) is the boundary condition for the standing oscillation. Then $\delta E_{x, \max }$ is regarded as $\delta E_{x}^{i}$. Note that the exact values of this ratio from the model and the equation cannot be compared to each other but the dependence can be discussed. Each lines is for a different value of $\bar{\Sigma}_{P 0}$ in the range from 1 to 1000 . The range of the conductivity variation is extremely large in order to show the dependence on the conductivity clearly. Note that the main magnetic field is assumed to be perpendicular to the ionosphere $\left(z_{0}=0\right)$. This figure reveals that $\delta E_{x, i s}$ is not determined only by $\bar{\Sigma}_{P 0}$. When $\hat{\Sigma}_{P}$ is much larger than the other terms in the denominator of Eq. (13), $\delta E_{x, i s} / \delta E_{x}^{i}$ is inversely proportional to $\hat{\Sigma}_{P}$. This condition where the ionospheric electric field is determined only by $\Sigma_{P}$ is referred to as the static condition (c.f., Poulter and Allan, 1985). When $k_{\|}^{A} \hat{\Sigma}_{H}^{2} / D$ is the most dominant term, on the other hand, $\delta E_{x, i s} / \delta E_{x}^{i}$ is proportional to $\Sigma_{H}{ }^{-2}$. From now on, the condition where the inductive electric field is effective is called the inductive condition (c.f., Yoshikawa and Itonaga, 1996).

It is interesting that the demarcation of the static and inductive conditions depends on the absolute value of the conductivity. In Fig. 4, the inductive field for $\bar{\Sigma}_{P 0}=1$ is effective when $\bar{\Sigma}_{H 0}$ is several times as large as $\bar{\Sigma}_{P 0}$ and that for $\bar{\Sigma}_{P 0}=1000$ is effective when $\bar{\Sigma}_{H 0}$ is as same as $\bar{\Sigma}_{P 0}$. From our estimation, the two terms have same absolute values when $\bar{\Sigma}_{H 0}=12.5 \bar{\Sigma}_{P 0}$ for $\bar{\Sigma}_{P 0}=1$ and $\bar{\Sigma}_{H 0}=1.05 \bar{\Sigma}_{P 0}$ for $\bar{\Sigma}_{P 0}=1000$. In this estimation, the values of $k_{x}=4$, $d=0.01, V_{A}=2.9$ are used. These relation is consistent with the results shown in Fig. 4.

From the discussion above, it is clear that the present numerical analysis yields the results consistent with previous works.

\subsection{Behaviors of standing Alfvén oscillations}

We investigate the behavior of standing Alfvén oscillations in the trapezoid model with various magnetosphere/ionosphere parameters. The second harmonic mode of standing Alfvén oscillations is considered because the magnetic perturbation is maximized in the equatorial plane. Hereafter, the following notations are used; $\delta B_{x, g r d}$ is the ground magnetic perturbation, $\delta B_{y, i s}$ is $\delta B_{y}$ just above the ionosphere and $\delta B_{y, e q}$ is that in the equatorial plane (Note that $\delta B_{y, e q}$ of the second harmonic Alfvén oscillation is the maximum $\left.\delta B_{y}\right)$.

3.2.1 Ionospheric conductivity The ratio of $\delta B_{x, g r d}$ to $\delta B_{y, i s}$ is presented in Fig. 5, which shows that the enhanced $\bar{\Sigma}_{H 0}$ results in the ionospheric shielding effect for the magnetic field perturbation as well as the reduction in the ionospheric electric field for the inductive condition. As the ground magnetic perturbation is proportional to the Hall current (which is a product of the Hall conductivity and the electric field), the ground magnetic perturbation is proportional to $\Sigma_{H}$ in the static condition and the inversely proportional in the inductive condition.

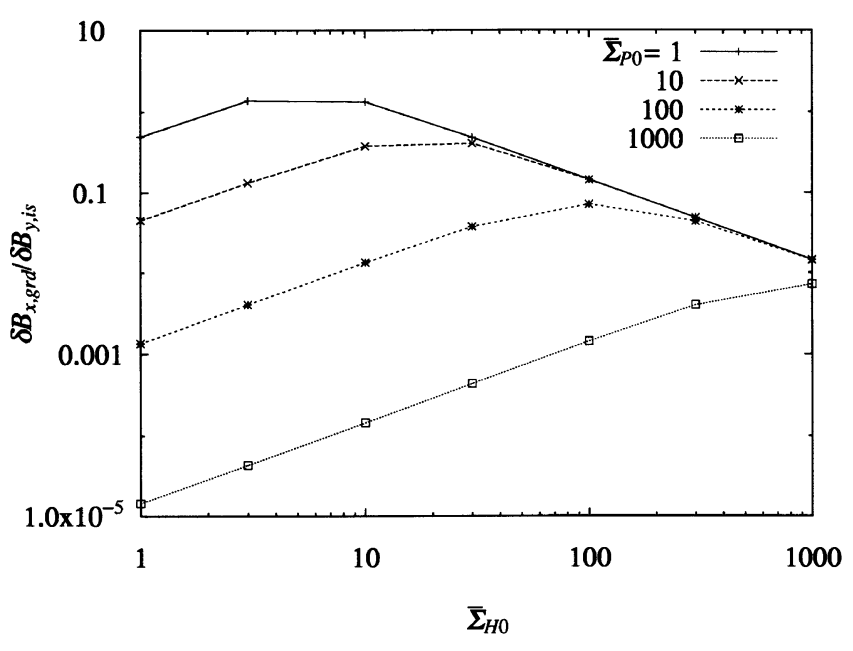

Fig. 5. The same as Fig. 4 for $\delta B_{x, g r d} / \delta B_{y, i s}$.

To clarify the relationship between the conductivities and the ground magnetic perturbations analytically, the following equation is derived using the same procedure as Eq. (13):

$$
\frac{\delta B_{x, g r d}}{\delta B_{y, i s}}=\frac{i k_{x}}{D \sinh \left(k_{x} d\right)} \frac{\hat{\Sigma}_{H}}{\hat{\Sigma}_{P}+k_{\|}^{A} \hat{\Sigma}_{H}^{2} / D} .
$$

Since the second term in the denominator of Eq. (14) is neglected for the static case, we have the following equation:

$$
\frac{\delta B_{x, \text { grd }}}{\delta B_{y, i s}} \simeq \frac{i k_{x}}{D \sinh \left(k_{x} d\right)} \frac{\hat{\Sigma}_{H}}{\hat{\Sigma}_{P}},
$$

which indicates that, when the ionospheric electric field is static, the Hall conductivity does not lead to a shielding effect for the ground magnetic perturbation. Equation (15) also demonstrates that the ground magnetic perturbation normalized to $\delta B_{y, i s}$ is proportional to $\hat{\Sigma}_{H} / \hat{\Sigma}_{P}$. If $\hat{\Sigma}_{P}$ is sufficiently large, $k_{\|}^{A} \hat{\Sigma}_{P}$ becomes the dominant term in $D$ and the ground magnetic perturbation is proportional to $\hat{\Sigma}_{H} / \hat{\Sigma}_{P}{ }^{2}$. On the other hand, in the inductive case, the second term of the denominator of Eq. (14) is dominant. Then Equation (14) becomes

$$
\frac{\delta B_{x, \text { grd }}}{\delta B_{y, i s}} \simeq \frac{i k_{x}}{k_{\|}^{A} \sinh \left(k_{x} d\right)} \frac{1}{\hat{\Sigma}_{H}} .
$$

From this equation, $\delta B_{x, g r d} / \delta B_{y, \text { is }}$ is seen to be inversely proportional to $\hat{\Sigma}_{H}$. This can be called the magnetic shielding effect due to ionospheric Hall current (Tamao, 1984; Fujita, 1985). This equation also shows that $\delta B_{x, \text { grd }} / \delta B_{y, \text { is }}$ in the inductive condition is independent of $\hat{\Sigma}_{P}$. Note that the two-step procedure of deriving the ground magnetic perturbation employed by Poulter and Allan (1985) and Allan (1995) did not reproduce the magnetic shielding effect due to the ionospheric Hall current.

Whether the ionospheric electric field is static or inductive controls the north-south asymmetry of the ground magnetic perturbation. In Fig. 6, the ratio of $\delta B_{x, g r d}$ in the northern hemisphere to that in the southern hemisphere $\left(\delta B_{x, g r d}(\mathrm{~N} / \mathrm{S})\right)$ is shown as a function of only $\bar{\Sigma}_{P 0}$ or $\bar{\Sigma}_{H 0}$ in the northern hemisphere. From now on, the notations of $(\mathrm{N})$ and $(\mathrm{S})$ are 


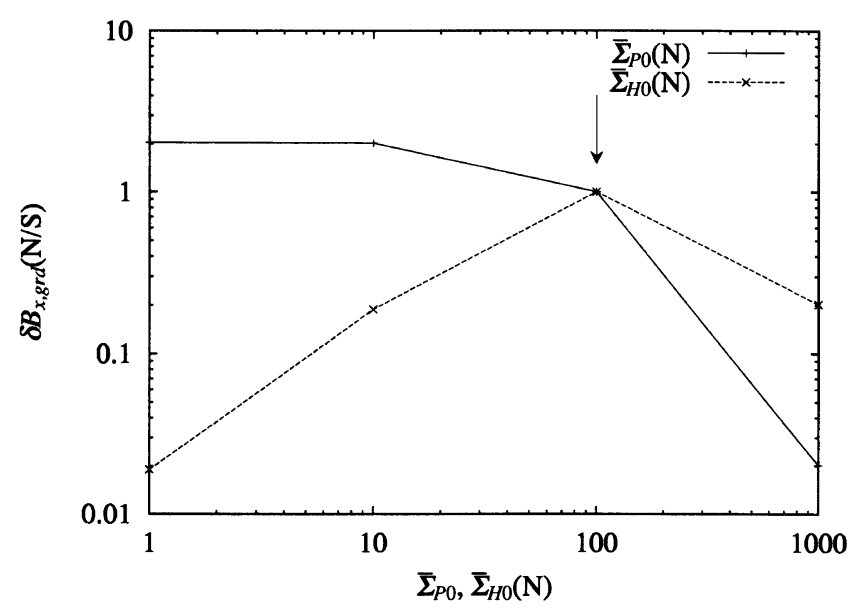

Fig. 6. $\delta B_{x, \text { grd }}(\mathrm{N} / \mathrm{S})$ as functions of $\bar{\Sigma}_{P 0}(\mathrm{~N})$ and $\bar{\Sigma}_{H 0}(\mathrm{~N})$. In this figure, $\bar{\Sigma}_{P 0}(\mathrm{~S})=\bar{\Sigma}_{H 0}(\mathrm{~S})=100$. The line with notation of " $\bar{\Sigma}_{P 0}(\mathrm{~N})$ " shows $\delta B_{x, g r d}(\mathrm{~N} / \mathrm{S})$ as a function of $\bar{\Sigma}_{P 0}(\mathrm{~N})$ with $\bar{\Sigma}_{H 0}(\mathrm{~N})=100$ and that of " $\bar{\Sigma}_{H 0}(\mathrm{~N})$ " shows $\delta B_{x, g r d}(\mathrm{~N} / \mathrm{S})$ as a function of $\bar{\Sigma}_{H 0}(\mathrm{~N})$ with $\bar{\Sigma}_{P 0}(\mathrm{~N})=100$. In both cases, $z_{0}=0$ and $V_{A, i s}=10$ in both hemispheres. An arrow in this figure shows the position where the conditions in the northern and southern ionospheres are same.

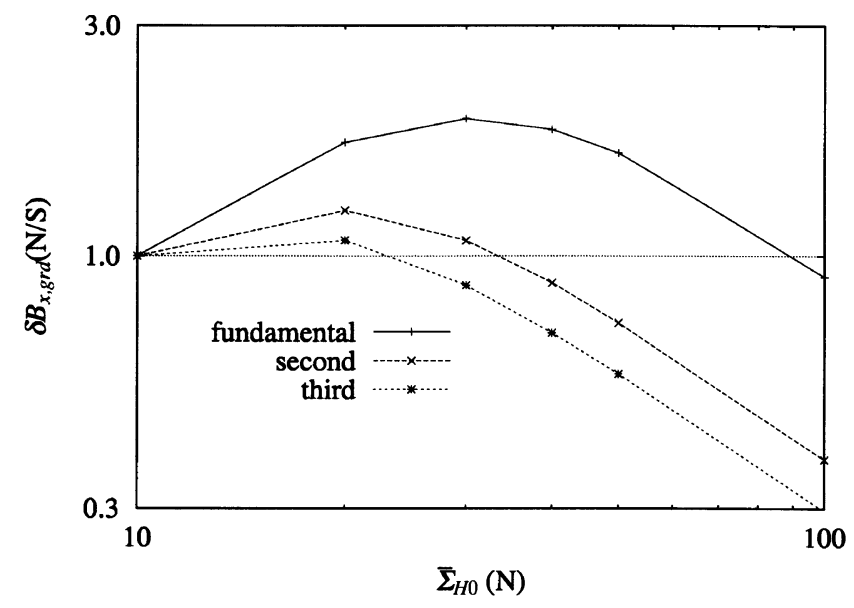

Fig. 7. $\delta B_{x, g r d}(\mathrm{~N} / \mathrm{S})$ of the first three harmonics of the standing Alfvén oscillation. The conductivities are $\bar{\Sigma}_{H 0}(\mathrm{~S})=\bar{\Sigma}_{P 0}(\mathrm{~N})=\bar{\Sigma}_{P 0}(\mathrm{~S})=10$.

referred to as components in the northern and southern hemispheres, respectively. To investigate the effect of varying $\bar{\Sigma}_{H 0}(\mathrm{~N})\left(\bar{\Sigma}_{P 0}(\mathrm{~N})\right)$, we set $\bar{\Sigma}_{P 0}(\mathrm{~N})=100\left(\bar{\Sigma}_{H 0}(\mathrm{~N})=100\right)$ and $\bar{\Sigma}_{H 0}(\mathrm{~S})=\bar{\Sigma}_{H 0}(\mathrm{~S})=100$. Both ionospheres are perpendicular to the main magnetic field. When $\delta E_{x, i s}(\mathrm{~N})$ is static (solid line for $\bar{\Sigma}_{P 0}(\mathrm{~N}) \rightarrow 1000$ or dashed line for $\left.\bar{\Sigma}_{H 0}(\mathrm{~N}) \rightarrow 1\right), \delta B_{x, \text { grd }}(\mathrm{N} / \mathrm{S})<1$ because $\delta B_{x, \text { grd }}$ is proportional to $\Sigma_{H} / \Sigma_{P}$. For the inductive condition, $\delta B_{x, \text { grd }}$ is larger in the hemisphere with smaller $\bar{\Sigma}_{H 0}$. Note that, when $\bar{\Sigma}_{P 0}(\mathrm{~N})<\bar{\Sigma}_{P 0}(\mathrm{~S})$ (solid line for $\bar{\Sigma}_{P 0}(\mathrm{~N}) \rightarrow 1$ ), $\delta B_{x, g r d}(\mathrm{~N})>1$ because of the shielding effect of $\Sigma_{P}$.

Next, let us consider the north-south asymmetry of the ground magnetic perturbations associated with the fundamental, second, and third harmonic standing oscillations. Figure 7 presents $\delta B_{x, g r d}(\mathrm{~N} / \mathrm{S})$ as a function of $\bar{\Sigma}_{H 0}(\mathrm{~N})$ for $10 \leq \bar{\Sigma}_{H 0} \leq 100$. It is interesting that, in this range of $\bar{\Sigma}_{H 0}, \delta B_{x, g r d}(\mathrm{~N})>\delta B_{x, \text { grd }}(\mathrm{S})$ for the fundamental oscillation, whereas this relation is reversed for the second and third
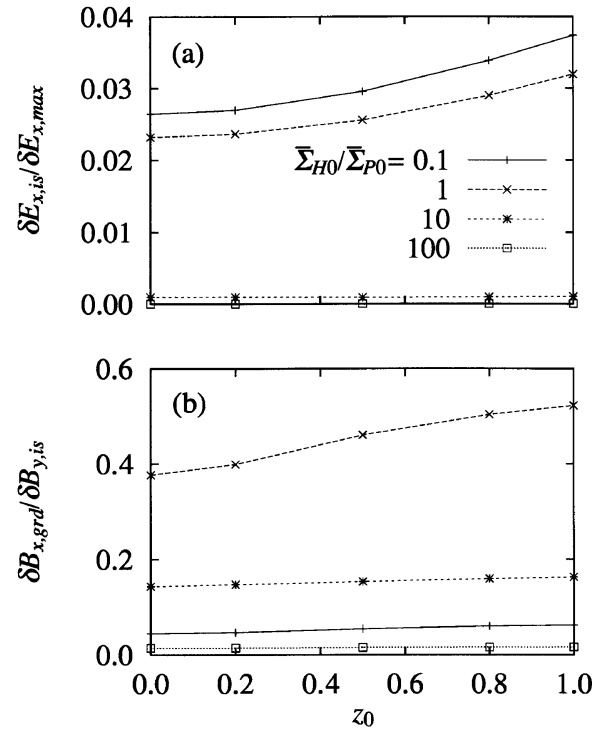

Fig. 8. $\delta E_{x, i s} / \delta E_{x, \max }$ (a) and $\delta B_{x, g r d} / \delta B_{y, i s}$ (b) as functions of $z_{0}$. The ratios in 4 cases of $\bar{\Sigma}_{H 0} / \bar{\Sigma}_{P 0}(=0.1,1,10$ and 100) are shown $\left(\bar{\Sigma}_{P 0}=10\right)$.

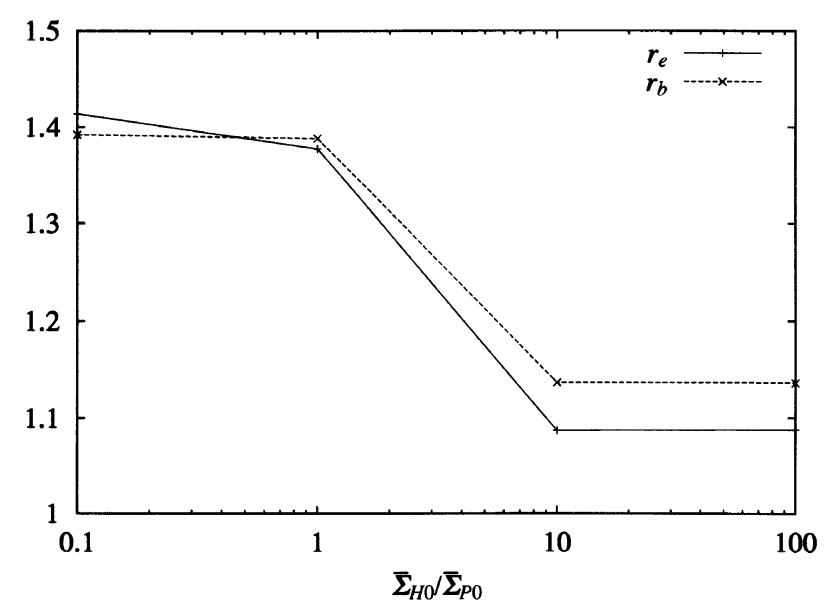

Fig. 9. $r_{e}$ (defined in Eq. (17) and $r_{b}$ (Eq. (18)) derived from the result of Fig. 8 as functions of $\bar{\Sigma}_{H 0} / \bar{\Sigma}_{P 0}$. The solid and dashed lines are $r_{e}$ and $r_{b}$, respectively.

harmonics. Let us explain this feature. In this range of $\bar{\Sigma}_{H 0}$, the ionospheric condition for the Alfvén oscillation is the transition between the static and inductive conditions. For the higher harmonics, the eigenfrequency becomes larger; this fact implies that the ionospheric electric field tends to be more inductive for the higher harmonics. Bearing in mind that the ionosphere makes the ground magnetic perturbation smaller in the inductive condition, we can understand that the ground magnetic perturbation is much reduced for the higher harmonics. The present result implies that, for conjugate observations, the north-south intensity relationship for longerperiod pulsations (e.g., Pc 5 pulsation) may be opposite to that of a shorter-period one (e.g., Pc 3). As shown later, in the daytime condition, $\Sigma_{H}$ is usually larger than $\Sigma_{P}$ and the Alfvén conductance $\left(\Sigma_{A}\right)$ is usually 10 times smaller than the ionospheric conductivity. Therefore, the north-south intensity ratio of the pulsations is likely dependent on frequency. 
3.2.2 Magnetic inclination Ionospheric transmission of the magnetic perturbation associated with standing Alfvén oscillations in the static condition exhibits the feature different from that in the inductive condition. Here, we investigate how the behavior of standing Alfvén oscillations is affected by magnetic inclination. The two ratios, $\delta E_{x, i s} / \delta E_{x, \max }$ and $\delta B_{x, \text { grd }} / \delta B_{y, i s}$, are plotted as functions of $z_{0}$ in Fig. 8 . The parameters used are $\bar{\Sigma}_{P 0}=10$ and $\bar{\Sigma}_{H 0} / \bar{\Sigma}_{P 0}=0.1,1,10$ and 100. From Fig. $8(\mathrm{a})$, we can see that $\delta E_{x, i s} / \delta E_{x, \max }$ increase with $z_{0}$ at least in the case of $\bar{\Sigma}_{H 0} \leq \bar{\Sigma}_{P 0}\left(\bar{\Sigma}_{H 0}=1\right.$ and 10).

To investigate the effect of the magnetic inclination in detail, Figure 9 illustrates the $\bar{\Sigma}_{H 0}$-dependence of $r_{e}$, which is defined as

$$
r_{e}=\left.\frac{\delta E_{x, i s}}{\delta E_{x, \max }}\right|_{z_{0}=1.0} /\left.\frac{\delta E_{x, i s}}{\delta E_{x, \max }}\right|_{z_{0}=0.0} .
$$

The parameter $r_{e}$ denotes control of the ionospheric electric field by the magnetic inclination. It is clear from Fig. 9 that the ionospheric electric field relative to the maximum electric field becomes larger along with increasing $z_{0}$ for $\bar{\Sigma}_{H 0} / \bar{\Sigma}_{P 0}=0.1$ and 1 (the static condition), whereas it does not vary so much for $\bar{\Sigma}_{H 0} / \bar{\Sigma}_{P 0}=10$ and 100 (the inductive condition). This feature is also seen in $\delta B_{x, \text { grd }} / \delta B_{y, \text { is }}$ shown in Fig. 8(b). Moreover, Figure 9 presents $r_{b}$ defined as

$$
r_{b}=\left.\frac{\delta B_{x, \text { grd }}}{\delta B_{y, i s}}\right|_{z_{0}=1.0} /\left.\frac{\delta B_{x, g r d}}{\delta B_{y, i s}}\right|_{z_{0}=0.0} .
$$

The variation of $r_{b}$ is essentially as same as $r_{e}$.

Let us consider further the physical implication of the numerical results. In the case that the ionospheric electric field is static, Allan and Knox (1979b) derived the boundary condition including the effect of the inclination when $\Sigma_{H}$ is neglected. They showed that $\hat{\Sigma}_{P}$ has the factor of $\sin I$ in the ionospheric boundary condition. As a result, the following relation is derived from Eq. (14):

$$
\frac{\delta B_{x, \text { grd }}}{\delta B_{y, i s}}=\frac{i k_{x}}{D \sinh \left(k_{x} d\right)} \frac{\hat{\Sigma}_{H}}{\hat{\Sigma}_{P} \sin I} .
$$

Using this relation, $r_{b}$ is analytically found to be $\sqrt{2}$. In Fig. $9, r_{b}$ is about 1.4 which is close to $\sqrt{2}$, for $\bar{\Sigma}_{H 0} / \bar{\Sigma}_{P 0}=$ 0.1 and 1 respectively. On the other hand, it is difficult to manipulate the effect of the inclination to $\Sigma_{H}$ in an analytical way. Therefore, we investigate the effect of the magnetic inclination on the behavior of standing Alfvén oscillations in the inductive condition using the numerical results. For the inductive condition, $\delta B_{x, g r d} / \delta B_{y, i s}$ in the case of $z_{0}=0$ led to Eq. (16), which shows that $\delta B_{x, \text { grd }} / \delta B_{y, \text { is }}$ is inversely proportional to $\hat{\Sigma}_{H}$. If $\hat{\Sigma}_{H}$ also has the same factor as $\hat{\Sigma}_{P}$, that is $\sin I, r_{b}$ must be equal to $\sqrt{2}$ respectively. However, $r_{b}$ for $\bar{\Sigma}_{H 0} / \bar{\Sigma}_{P 0}=10$ and 100 is about 1.1 , which shows that the variation of the effect of $\bar{\Sigma}_{H 0}$ is smaller than that of $\bar{\Sigma}_{P 0}$. Consequently, the effect of the inclination is not significant for the inductive condition.

The above discussion suggests smaller north-south asymmetry in the ground magnetic field due to the north-south asymmetry in the inclination for the inductive condition. In Fig. 10, $\delta B_{x, g r d}(\mathrm{~N} / \mathrm{S})$ for the cases $\bar{\Sigma}_{H 0}=10=\bar{\Sigma}_{P 0}$ (almost the static condition) and $\bar{\Sigma}_{H 0}=100=10 \bar{\Sigma}_{P 0}$ (the

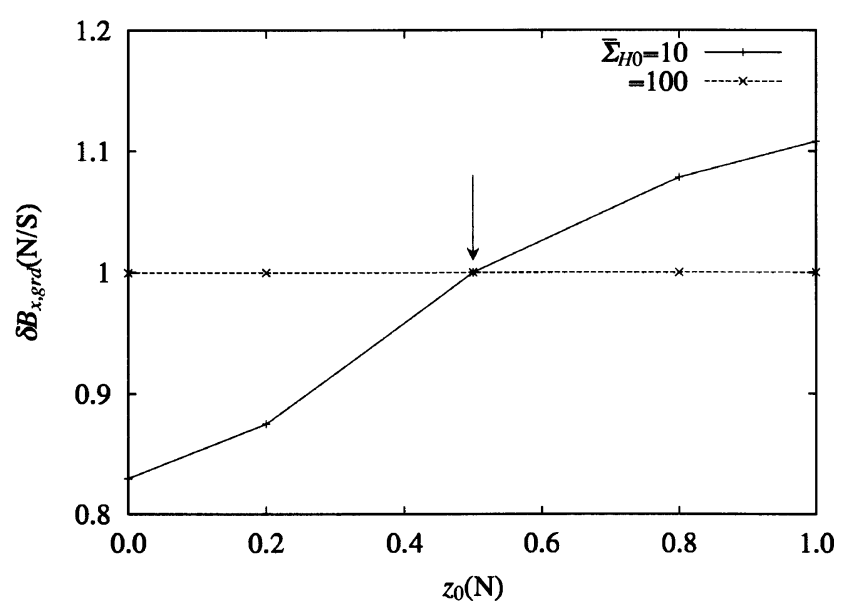

Fig. 10. $\delta B_{x, g r d}(\mathrm{~N} / \mathrm{S})$ as a function of $z_{0}(\mathrm{~N})$. In this figure, $z_{0}(\mathrm{~S})=0.5$ and $\bar{\Sigma}_{P 0}(\mathrm{~N})=\bar{\Sigma}_{P 0}(\mathrm{~S})=10 . \bar{\Sigma}_{H 0}(\mathrm{~N})$ and $\bar{\Sigma}_{P 0}(\mathrm{~S})$ is 10 (solid line) and 100 (dashed line). The conditions of both ionosphere are same except the inclination. But they are same where the arrow points.
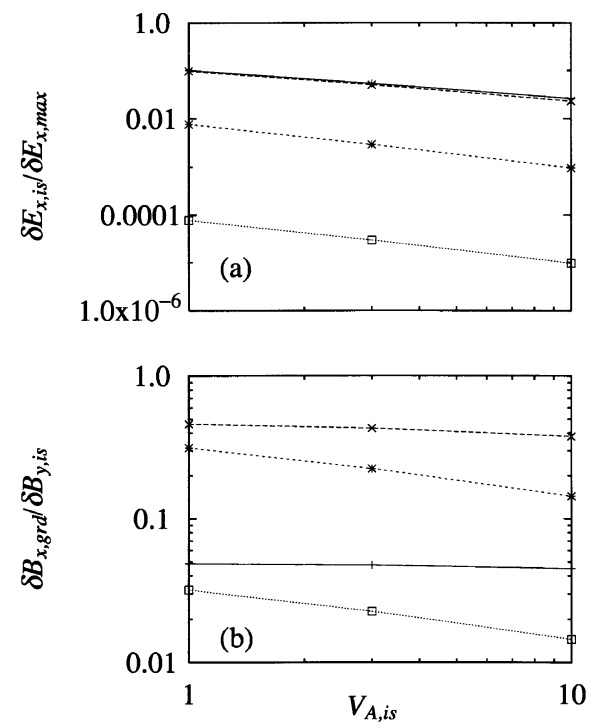

Fig. 11. $\delta E_{x, i s} / \delta E_{x, \max }$ (a), $\delta B_{x, \text { grd }} / \delta B_{y, \text { is }}$ (b) as functions of $V_{A, i s}$. The values of the conductivities are as same as Fig. 8 .

inductive condition) are shown with $z_{0}(\mathrm{~S})=0.5$. The ionospheric conductivity is north-south symmetric and the only variable is $z_{0}(\mathrm{~N})$. For the static condition $\left(\bar{\Sigma}_{H 0}=10\right)$, $\delta B_{x, \text { grd }}(\mathrm{N} / \mathrm{S})$ is nearly proportional to $\sin I$. Since the magnetic inclination $(I)$ reduces $\Sigma_{P}$ by $\sin I$, the effective $\Sigma_{P}(=$ $\Sigma_{P} \sin I$ ) is larger (or smaller) in the northern hemisphere for $z_{0}(\mathrm{~N})<z_{0}(\mathrm{~S})\left(\right.$ or $\left.z_{0}(\mathrm{~N})>z_{0}(\mathrm{~S})\right)$. As explained above, larger $\bar{\Sigma}_{P 0}$ reduces the ground magnetic perturbation in the static condition. On the other hand, for the inductive condition $\left(\bar{\Sigma}_{H 0}=100=10 \bar{\Sigma}_{P 0}\right)$, the inclination does not affect the transmission of the magnetic perturbation across the ionosphere. Thus, the inclination effectively controls the behavior of standing Alfvén oscillations only in the static condition.

3.2.3 Equatorial plane-ionosphere $V_{A}$ ratio We investigate how the behavior of standing Alfvén oscillations changes according to variation in $V_{A, i s} / V_{A, e q}\left(V_{A, i s}\right.$ and 


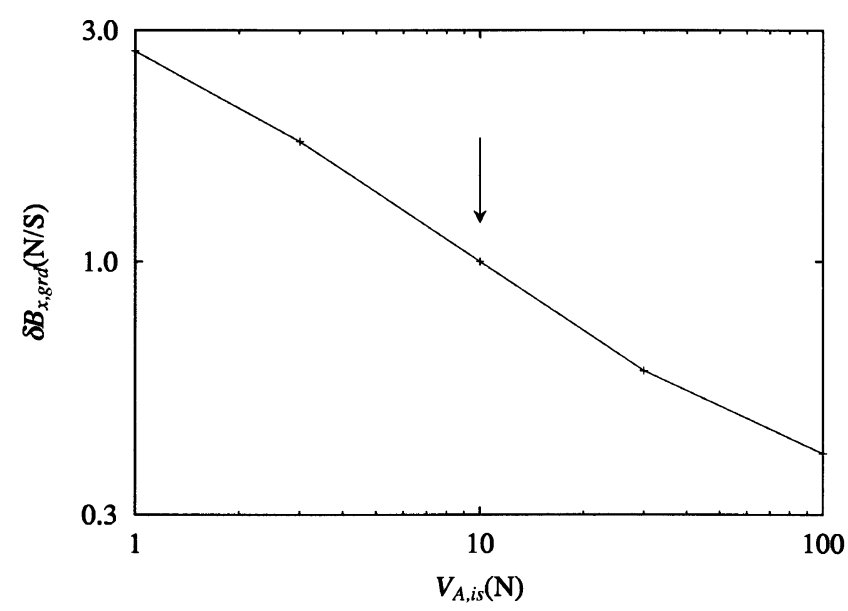

Fig. 12. $\delta B_{x, g r d}(\mathrm{~N} / \mathrm{S})$ as a function of $V_{A, i s}(\mathrm{~N}) . \quad V_{A, i s}(\mathrm{~S})=10$, $z_{0}(\mathrm{~N}, \mathrm{~S})=0$ and $\bar{\Sigma}_{P 0}(\mathrm{~N}, \mathrm{~S})=\bar{\Sigma}_{H 0}(\mathrm{~N}, \mathrm{~S})=10$.

$V_{A, e q}$ are the Alfvén speeds in the ionosphere and in the equatorial plane on the field line concerned). As noted before, since the trapezoid model has straight magnetic field lines, the flux tube convergence that appears in the real magnetosphere is not considered here. Therefore, we consider only the effect of partial reflection of the Alfvén wave due to the increase in $V_{A}$ along the field line.

Figure 11 shows $\delta E_{x, i s} / \delta E_{x, \max }$ and $\delta B_{x, \text { grd }} / \delta B_{y, \text { is }}$ as functions of $V_{A, i s}$ for the same conductivity values used in Fig. 8, but the main magnetic field is perpendicular to the ionosphere. Since $\bar{\Sigma}_{P 0}(=10)$ is larger than the Alfvén conductance ( $=1$ for $V_{A, i s}=1$ and 0.42 for $V_{A, i s}=10$ ), $\delta E_{x}$ has a node and $\delta B_{y}$ has an anti-node in the ionosphere. It is evident from Fig. 11(a) that $\delta E_{x, i s} / \delta E_{x, \max }$ decreases with increasing $V_{A, i s}$. This feature can be explained by the partial reflection of the Alfvén wave in the magnetosphere where $V_{A}$ increases as one moves toward the ionosphere along the field line. Figure 11(b) shows the decreases of $\delta E_{x, i s} / \delta E_{x, \max }$ and $\delta B_{x, \text { grd }} / \delta B_{y, \text { is }}$ with increasing $V_{A, i s}$ are dependent on $\bar{\Sigma}_{H 0}$. It is evident from Fig. 11(b) that $\delta B_{x, g r d} / \delta B_{y, \text { is }}$ is almost independent of $V_{A, i s}$ for $\bar{\Sigma}_{H 0} / \bar{\Sigma}_{P 0} \leq 1$ (the static condition) and that it decreases with increasing $V_{A, i s}$ for $\bar{\Sigma}_{H 0} / \bar{\Sigma}_{P 0} \geq 10$ (the inductive condition). For the static condition, the first term of the denominator in Eq. (14) is dominant, while for the inductive condition, the second term (which is dependent on the frequency) is dominant. In fact, the eigenfrequency of standing Alfvén oscillations is affected by $V_{A, i s}$ and thus the ground magnetic perturbation is affected by $V_{A, i s}$ as well.

We now investigate the asymmetry of the ground magnetic perturbations invoked by the north-south asymmetry of the field-aligned variation in $V_{A}$. The present model assumes the field-aligned profile of $V_{A}$ defined by Eq. (3). Thus, the larger $V_{A, i s}$ indicates that $V_{A}$ becomes larger as one moves toward the ionosphere. Figure 12 shows $\delta B_{x, g r d}(\mathrm{~N} / \mathrm{S})$ as a function of $V_{A, i s}(\mathrm{~N})$ in the case where $V_{A, i s}(\mathrm{~S})=10$ where $\bar{\Sigma}_{P 0}=$ $\bar{\Sigma}_{H 0}=10$ in both ionospheres. The main magnetic field is perpendicular to the ionosphere. Since the ionospheric conductivities in both hemispheres are the same, the variation of $\delta B_{x, g r d}(\mathrm{~N} / \mathrm{S})$ with $V_{A, i s}(\mathrm{~N})$ is the same whether the ionospheric electric field is static or inductive. Here only the static case is treated. This figure indicates that $\delta B_{x, g r d}$ in the hemisphere with larger $V_{A, i s}$ is smallest. This feature is interpreted as follows; first, partial reflection of the Alfvén wave due to the increase in $V_{A}$ along the field line reduces the electric field perturbation of the Alfvén wave moving toward the ionosphere. Then the larger $V_{A, i s}$ becomes, the smaller will be the ionospheric electric field perturbation associated with the Alfvén wave. Thus, the ground magnetic perturbation, which is the product of the ionospheric electric field and $\Sigma_{H}$, is smallest in the hemisphere with larger $V_{A, i s}$.

Note that asymmetry of $V_{A}$ in the actual magnetosphere is produced by the asymmetries of the main magnetic field intensity and the plasma mass density. The former is associated with convergence of the magnetic flux towards the ionosphere. This convergence may yield an intensification of the electromagnetic field perturbation in the hemisphere with the larger main magnetic field intensity. The flux convergence effect introduces a bias in the north-south asymmetry of the ground magnetic perturbations independent of season. On the other hand, the partial reflection effect yields a smaller electromagnetic field perturbation in the hemisphere with larger $V_{A}$. When the asymmetry in $V_{A}$ is invoked by the plasma mass density asymmetry, the partial reflection effect introduces the seasonal variation of the north-south asymmetry. Therefore, it is possible to compare the seasonal variation of the asymmetry derived in the present numerical analysis to that observed.

\section{The Ground Magnetic Perturbations Associ- ated with Standing Alfvén Oscillations in the Realistic Model}

Using the realistic values of ionospheric conductivity, magnetic inclination, the height of the ionosphere $(d)$ together with a reasonable equatorial plane-ionosphere ratio of $V_{A}$, we evaluate the asymmetry of the ground magnetic perturbations at the conjugate points. Note that the present model employs straight magnetic field lines, which differ from the actual dipole magnetic field. In spite of this difference, we can find important features of the north-south asymmetry of the ground magnetic perturbations by using the present model calculation. We investigate the latitudinal and longitudinal dependence of asymmetries at the conjugate points for three $L$ values (2, 4 and 6) and 4 magnetic longitudes $\left(\Phi=0^{\circ}\right.$, $90^{\circ}, 180^{\circ}$ and $270^{\circ}$ ). In obtaining the coordinates of the conjugate points, the dipole coordinates, which is measurably different from the geomagnetic coordinate, is used in order to clear reasons of the longitudinal dependence. However, we can investigate the dependence because the value of the asymmetry is not mentioned.

\subsection{Assignment of the realistic physical values}

As regards the Alfvén speed in the ionosphere $\left(V_{A, i s}\right)$, the north-south asymmetries of the plasma mass density and the magnetic field intensity should be taken into consideration. We use IRI90 (Bilitza, 1990) for the estimation of the plasma density. Bearing in mind that standing Alfvén oscillations with long wavelength feel $V_{A}$ averaged along the field line, the ion density averaged in the region from 80 to $2000 \mathrm{~km}$ is assumed to contribute to $V_{A, i s}$. IGRF95 (IAGA, 1995) is used for the magnetic field intensity. Then, $V_{A, i s}(\mathrm{~N})$ and 
Table 1. The eigenfrequencies of standing Alfvén oscillation on three $L$-shells used for the derivation of the Alfvén speed in the equatorial plane.

\begin{tabular}{ccl}
\hline$L$ & Freq. (mHz) & Reference \\
\hline 6 & 6.0 & Samson and Rostoker (1972) \\
4 & 10.7 & Samson and Rostoker (1972) \\
2 & 27.5 & Ziesolleck et al. (1993) \\
\hline
\end{tabular}

$V_{A, i s}(\mathrm{~S})$ are given by

$$
\begin{aligned}
V_{A, i s}(\mathrm{~N})= & V_{A, e q} L \sqrt{1+3 \cos \theta_{L}} \frac{2 B_{0}(\mathrm{~N})}{B_{0}(\mathrm{~N})+B_{0}(\mathrm{~S})} \\
& \times \frac{\sqrt{\rho(\mathrm{N})}+\sqrt{\rho(\mathrm{S})}}{2 \sqrt{\rho(\mathrm{N})}} \\
V_{A, i s}(\mathrm{~S})= & V_{A, e q} L \sqrt{1+3 \cos \theta_{L}} \frac{2 B_{0}(\mathrm{~S})}{B_{0}(\mathrm{~N})+B_{0}(\mathrm{~S})} \\
& \times \frac{\sqrt{\rho(\mathrm{N})}+\sqrt{\rho(\mathrm{S})}}{2 \sqrt{\rho(\mathrm{S})}}
\end{aligned}
$$

where $\theta_{L}, B_{0}$, and $\rho$ are respectively the colatitude of the foot point of an $L$-shell, the total magnetic field in the ionosphere, and the plasma mass density. The variation of $V_{A}$ along the field line is proportional to $z^{2}$ as shown in Eq. (3).

IRI90 and CIRA72 (Rees, 1988) are used for calculation of the height-integrated ionospheric conductivity. This integration range of 80-180 km includes the peak in the height profile of the ionospheric conductivity. The values of Pedersen and Hall conductivities at the upper boundary become smaller than one third of each peak values. We also employ 100 as the sunspot number because this value is roughly averaged one. The local time is assigned as $12 \mathrm{LT}$ because Pc pulsations are mainly observed in the daytime. To investigate the seasonal dependence, the conductivities in the equinoctial and solstitial months (March, June, September and December) are calculated.

To obtain the ionospheric conductivity normalized to the Alfvén conductance, we need values of the Alfvén speed in the equatorial plane $\left(V_{A, e q}\right)$ for each $L$-shell. These values are derived from comparisons between the observational eigenfrequencies as listed in Table 1 and calculated ones. Bearing in mind that the eigenfrequency of standing Alfvén oscillations depends on the ionospheric conductivity and the Alfvén conductance, we need to specify these two conductivities in the observations. Samson and Rostoker (1972) analyzed Pc 4 and Pc 5 events along a Canadian meridian $(L \geq 4)$ during northern summers of two years. The conductivity of the southern conjugate ionosphere is probably smaller than the Alfvén conductance of the field line at $L=6$ because the ionosphere is located in the dark hemisphere Actually, the present procedure yields an ionospheric conductivity of $0.16 \mathrm{~S}$ and an Alfvén conductance of $0.35 \mathrm{~S}$. Thus, standing Alfvén oscillations can be regarded as having an anti-node of the electric field perturbation in the southern ionosphere. On the contrary, in the northern ionosphere, the conductivity becomes larger than the Alfvén conductance. This implies that the oscillation has a node of the electric field perturbation in the ionosphere. Thus, the fundamental standing Alfvén oscillation in $L=6$ may become a quarterwave mode (Allan and Knox, 1979a). In order to obtain $V_{A, e q}$ at $L=6$, we calculate the eigenfrequency using ionospheric conductivities larger than the Alfvén conductance (e.g., $\bar{\Sigma}_{P 0}=\bar{\Sigma}_{H 0}=1000$ ) in the northern ionosphere and conductivities smaller than it (e.g., $\bar{\Sigma}_{P 0}=\bar{\Sigma}_{H 0}=0$ ) in the southern ionosphere. On $L$-shells of $L=2$ and 4 , standing Alfvén oscillations seem to have nodes of the electric field perturbations in the both ionospheres. At these $L$-shells, the eigenfrequencies calculated with large ionospheric conductivities in the both ionospheres are compared with the observed frequencies. As a result, $V_{A, e q}$ thus evaluated are $1090 \mathrm{~km} / \mathrm{s}, 990 \mathrm{~km} / \mathrm{s}$ and $1250 \mathrm{~km} / \mathrm{s}$ for $L=2,4$ and 6 , respectively.

The height of the ionosphere $(d)$ is normalized to the length of the field line. Since this height is $100 \mathrm{~km}$, the values of $d$ are $0.0176,0.0065$ and 0.004 for $L=2,4$ and 6 , respectively. The inclinations are also obtained from IGRF95.

\subsection{Characteristic features of asymmetry of the conju- gate ground magnetic perturbations}

First, the dependence on $L$-value of $\delta B_{x, g r d}(\mathrm{~N} / \mathrm{S})$ associated with the three harmonics on the $L$-value is investigated. Figure 13 shows $\delta B_{x, g r d}(\mathrm{~N} / \mathrm{S})$ at three conjugate points $(L=2,4,6)$ at $270^{\circ}$ longitude. From this figure, it can be seen that $\delta B_{x, g r d}(\mathrm{~N} / \mathrm{S})$ of the second and third harmonics is smaller in the summer hemisphere than in the winter hemisphere and while its value for the fundamental mode is almost constant.

This feature gives us a hint in specifying the interaction between the ionosphere and standing Alfvén oscillations, as to whether the static condition or the inductive one is appropriate. For the inductive condition, $\delta B_{x, \text { grd }} / \delta B_{y, i s}$ is proportional to $1 / \Sigma_{H}$. As $\Sigma_{H}$ in the summer is larger than that in the winter, $\Sigma_{H}(\mathrm{~N} / \mathrm{S})$ reaches its maximum in June and its minimum in December. This variation of $\Sigma_{H}$ yields the seasonal variation of $\delta B_{x, g r d}(\mathrm{~N} / \mathrm{S})$; it is larger in winter and smaller in summer. This variation is consistent to that of $\delta B_{x, g r d}$ of the second and third harmonics shown in Fig. 13. In the static condition, on the other hand, $\delta B_{x, g r d}$ is proportional to $\Sigma_{H} / \Sigma_{P}$. As $\Sigma_{H} / \Sigma_{P}$ is almost constant during the year, $\delta B_{x, \text { grd }}$ does not vary so much. Consequently, only the ionospheric electric field associated with the fundamental mode is static, whereas, those of the higher harmonics are inductive.

The results described above indicate that the two-step procedure of evaluating the ground magnetic perturbation (Allan, 1995) is valid for the fundamental mode oscillation because the ionospheric electric field is almost static. As for the higher harmonics, we need to treat the interaction be-

Table 2. The ratios of the amplitudes of pulsations at two conjugate pairs located on $210^{\circ} \mathrm{MM}$ chain.

\begin{tabular}{ccc}
\hline & KOT/MCQ (High Lat.) & MSR/BRV (Low Lat.) \\
\hline Pc 3 & winter $>$ summer & north $>$ south \\
Pc 4-5 & north $>$ south & - \\
\hline
\end{tabular}



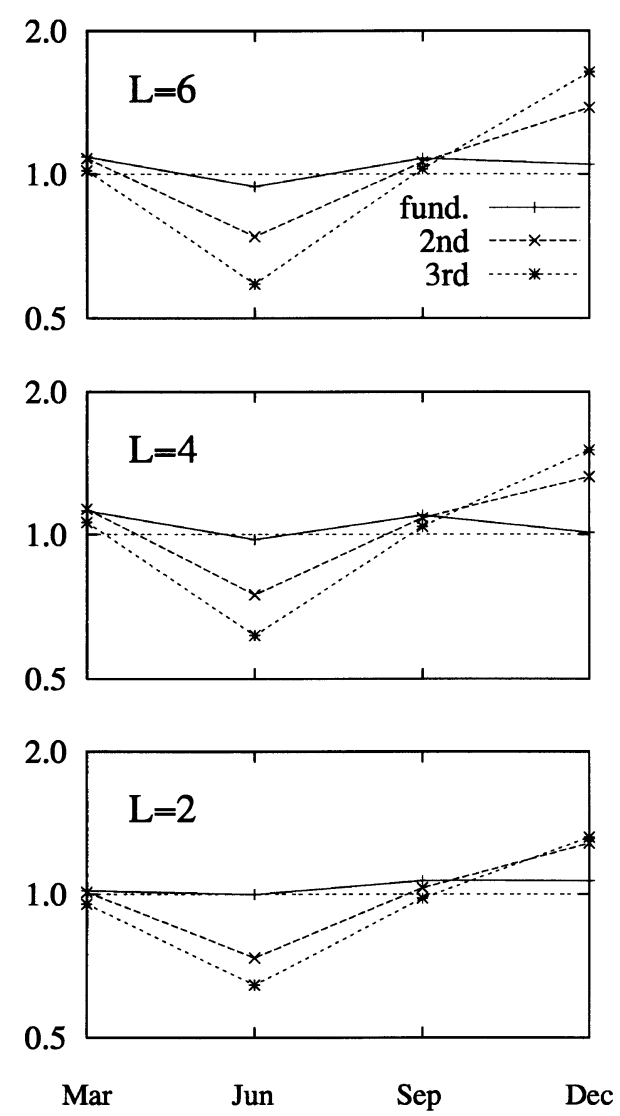

Fig. 13. The dependence of seasonal variations of $\delta B_{x, g r d}(\mathrm{~N} / \mathrm{S})$ on the $L$-value. The results of this figure are calculated with realistic parameters in equinoctial and solstitial seasons. The solid, dashed and dotted lines show the fundamental, second and third harmonics respectively. The top, middle and bottom panel are for $L=6,4$ and 2, respectively.

tween the MHD wave and the anisotropic conducting ionosphere in a self-consistent manner.

Next, the dependence of the $\delta B_{x, g r d}(\mathrm{~N} / \mathrm{S})$ on the magnetic longitude is investigated. Figure 14 shows seasonal variations of $\delta B_{x, g r d}(\mathrm{~N} / \mathrm{S})$ at the conjugate points for $L=6$. The dependence on the harmonics is the same as shown in Fig. 13 except for June at $\Phi=0^{\circ}$ and December at $180^{\circ}$. The reason for this exception will be explained later. The seasonal variations of $\delta B_{x, g r d}(\mathrm{~N} / \mathrm{S})$ at $\Phi=90^{\circ}$ are similar to that at $270^{\circ}$, while those at $\Phi=0^{\circ}$ are different from those at $180^{\circ}$. Particularly, at the solstice, $\delta B_{x, g r d}(\mathrm{~N} / \mathrm{S})$ at $\Phi=0^{\circ}$ reach a minimum, while at $\Phi=180^{\circ}$ it reaches a maximum. Its values at $\Phi=90^{\circ}$ and $270^{\circ}$ are somewhere in between.

It seems curious that the seasonal variation of $\delta B_{x, g r d}(\mathrm{~N} / \mathrm{S})$ depends on longitude. This is attributed to the difference between the geographic and geomagnetic coordinates. That is, the conjugate points at $\Phi=90^{\circ}$ and at $270^{\circ}$ are at the same distance from the geographic equator. On the other hand, at $\Phi=0^{\circ}$ and at $180^{\circ}$, the conjugate points shift southward and northward, respectively. In June, $\Sigma_{H}(\mathrm{~N} / \mathrm{S})$ increases at $\Phi=0^{\circ}$ and decreases at $\Phi=180^{\circ}$. As $\delta B_{x, \text { grd }}$ of the second and third modes are proportional to $1 / \Sigma_{H}$ (see Eq. (16)). $\delta B_{x, g r d}$ at $\Phi=0^{\circ}$ is smaller and that at $\Phi=180^{\circ}$ is larger.

The seasonal variation of the asymmetry for the fundamental mode exhibits anomalous behavior at $\Phi=0^{\circ}$ and $180^{\circ}$. The exceptionally enhanced asymmetry for the fundamental mode (at $L=6$ ) is explained by the difference of the ionospheric conductivities in the conjugate ionospheres. The difference in ionospheric conductivities is due to the fact that solar illumination is best ordered in geographic coordinate. In magnetic coordinate, conjugate points are therefore not expand to the same solar illumination and thus feature different ionospheric conductivities. For example, in June the ionosphere experiences almost daytime conditions in the northern hemisphere and nighttime conditions in the southern hemisphere at $\Phi=0^{\circ}$. Therefore, the asymmetry of the ionospheric conductivities between the conjugate points at $\Phi=0^{\circ}$ is quite enhanced. Note that the conductivity in the southern ionosphere falls to less than the Alfvén conductance, whereas, that in the northern ionosphere remains larger than the Alfvén conductance. Therefore, the electric field associated with standing Alfvén oscillations has an anti-node in the southern ionosphere and a node in the northern ionosphere (i.e., quarter wave) and $\delta B_{x, g r d}(\mathrm{~S})$ becomes much smaller than $\delta B_{x, g r d}(\mathrm{~N})$. Thus $\delta B_{x, g r d}(\mathrm{~N} / \mathrm{S})$ of the fundamental mode becomes largest in June at $\Phi=0^{\circ}$. Note that the anomalous seasonal variation of the asymmetry does not appear at $L=4$. This is because the ionospheric conductivity is not so small as to make the mode in the ionosphere an anti-node.

\subsection{Comparison with observations}

There are several works that have considered the conjugate asymmetry of the ground magnetic perturbations (e.g., Saito et al., 1989 and reference therein). As described before, only variations can be treated in comparing the numerical results with observations. Here, we compare the present results with the data acquired in the ground-based magnetic observation campaign called the $210^{\circ} \mathrm{MM}$ observation (Yumoto et al., 1992). In this campaign, the H-components of Pc pulsations at conjugate pairs were analyzed in order to investigate the north-south asymmetries of the Pc 3-5 power at high ( $L \sim 5.4)$ and low $(L \sim 1.6)$ latitudes. These data were obtained in the northern hemisphere summer (1993/6-8) and northern hemisphere winter (1993/12-1994/2) at Kotzebue (KOT: $66.88^{\circ}, 197.40^{\circ}$ geographic), Macquarie Isle. (MCQ: $\left.-54.50^{\circ}, 158.95^{\circ}\right)$, Moshiri (MSR: $44.37^{\circ}, 142.27^{\circ}$ ) and Birdsville (BRV: $\left.-25.54^{\circ}, 139.21^{\circ}\right)$. As shown in Table 2, a preliminary analysis of the $210^{\circ} \mathrm{MM}$ data reveals that $\mathrm{Pc}$ 3 power at low latitudes and Pc 4-5 power at high latitudes are larger in the hemisphere with a smaller main magnetic field strength (KOT > MCQ, MSR > BRV). It is also found that Pc 3 power at higher latitudes is larger in the winter hemisphere than in the summer hemisphere.

Let us compare the observations with our numerical results. Since the observations present only qualitative results on the north-south asymmetry, we discuss the asymmetry by comparing the ionospheric and magnetospheric parameters in both hemispheres. In Table 3, we show parameters of conjugate pairs estimated as described in Section 4.1. Let us first look at the high-latitude observation (KOTMCQ pair). As the magnetic inclination at KOT is almost the same as MCQ (The ratio of $\sin I$ at KOT to at MCQ is $0.991)$, the asymmetry of $\delta B_{x, g r d}$ is caused by differences in the conductivity and the Alfvén speed. At those latitudes, the longer-period pulsations (Pc 4-5) are considered 
Table 3. Ratios of the values of parameters in the northern hemisphere to those in the southern hemisphere (N/S) at the $210^{\circ} \mathrm{MM}$ stations estimated using realistic models (IGRF, IRI, CIRA).

\begin{tabular}{cclccc}
\hline Station pair & $\sin I$ & northern season & $\Sigma_{H}$ & $\Sigma_{H} / \Sigma_{p}$ & $V_{A}$ \\
\hline \multirow{2}{*}{ KOT/MCQ } & \multirow{2}{*}{0.991} & summer & 1.97 & 0.98 & 0.87 \\
& & winter & 0.33 & 1.07 & 0.99 \\
\hline \multirow{2}{*}{ MSR/BRV } & \multirow{2}{*}{1.01} & summer & 1.24 & 1.00 & 0.93 \\
& & winter & 0.70 & 0.91 & 1.04 \\
\hline
\end{tabular}
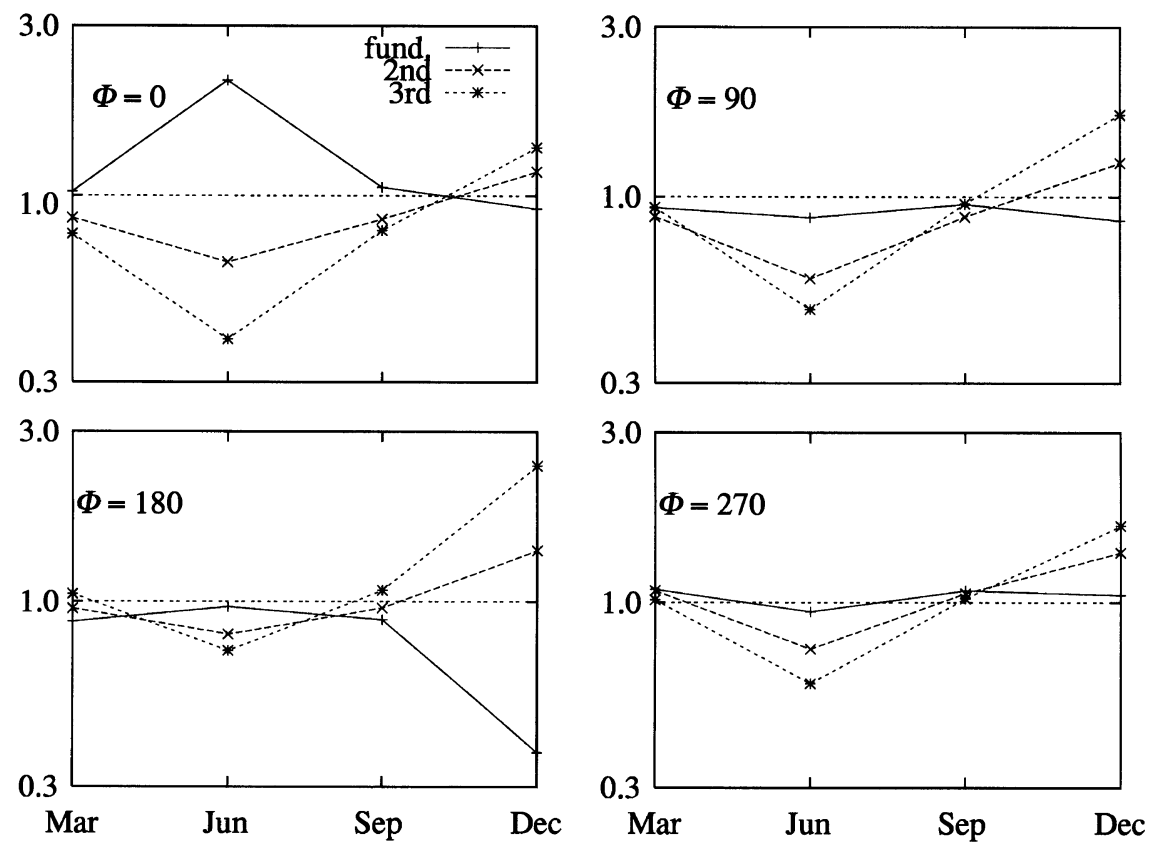

Fig. 14. The dependence of seasonal variations of $\delta B_{x, g r d}(\mathrm{~N} / \mathrm{S})$ on the magnetic longitude $\left(=0^{\circ}, 90^{\circ}, 180^{\circ}\right.$ and $\left.270^{\circ}\right)$. The way of the estimation of the parameters are as same as Fig. 13.

to be the fundamental mode oscillations, which have a static electric field in the ionosphere. For static conditions, as described before, $\delta B_{x, \text { grd }}$ is proportional to $\Sigma_{H} / \Sigma_{P}$ when $\Sigma_{P}>\Sigma_{A}$. From our estimation, $\Sigma_{P}$ at KOT and MCQ is larger than $\Sigma_{A}$ in both hemispheres. $\Sigma_{H} / \Sigma_{P}$ (KOT/MCQ) is proportional to the asymmetry of $\delta B_{x, \text { grd }}(\mathrm{KOT} / \mathrm{MCQ})$ due to the ionospheric conductivity. As $\delta B_{y, i s}$ decreases with $V_{A, i s}$, moreover, $V_{A, \text { is }}$ (KOT/MCQ) shows that the asymmetry of $\delta B_{x, g r d}$ is due to the Alfvén speed. Thus both parameters are considered to influence asymmetry of $\delta B_{x, \text { grd }}$. Besides, $V_{A, i s}(\mathrm{KOT} / \mathrm{MCQ})$ is smaller than 1 in both seasons $(0.87$ in northern summer and 0.99 in northern winter). These calculations suggest $\delta B_{x, g r d}(\mathrm{KOT} / \mathrm{MCQ})>$ 1 in the northern winter case. In the northern summer, $\Sigma_{H} / \Sigma_{P}(\mathrm{KOT} / \mathrm{MCQ})<1$ and $V_{A, i s}(\mathrm{KOT} / \mathrm{MCQ})<1$ but $V_{A, i s}(\mathrm{KOT} / \mathrm{MCQ}) \ll \Sigma_{H} / \Sigma_{P}(\mathrm{KOT} / \mathrm{MCQ})$. Thus, this result indicates $\delta B_{x, \text { grd }}(\mathrm{KOT} / \mathrm{MCQ})>1$ and therefore our calculations are consistent with observations. For the higher harmonics ( $\mathrm{Pc} 3$ ) featuring the inductive ionospheric electric field, $\delta B_{x, \text { grd }}$ may be larger in the winter hemisphere because smaller $\Sigma_{H}$ in the winter hemisphere at KOT and MCQ reduces the shielding effect due to the ionospheric Hall conductivity. The asymmetry in $V_{A}$ which is smaller than that for $\Sigma_{H}$ is not significant. Thus, in both cases of longer- and shorter-period oscillations, our study is consistent with observations at higher latitudes obtained using the $210^{\circ} \mathrm{MM}$ chain. This suggests that the convergence of the magnetic flux tubes toward the ionosphere has no significant influence in the high latitude case.

Finally, let us consider the asymmetry observed at lower latitudes during the $210^{\circ} \mathrm{MM}$ observation campaign. Pc 3 corresponds to the fundamental mode. $\Sigma_{H} / \Sigma_{P}(\mathrm{MSR} / \mathrm{BRV})$ and $V_{A, i s}(\mathrm{MSR} / \mathrm{BRV})$ are 1.00 and 0.93 in the northern summer and 0.91 and 1.04 in the northern winter, respectively. Note that the inclinations of the magnetic field at both stations are also almost the same. From these values, our calculations suggest that $\delta B_{x, g r d}(\mathrm{MSR} / \mathrm{BRV})$ of the fundamental mode is larger than 1 in the northern summer and smaller than 1 in the northern winter. This result is consistent with the observations in the northern summer, whereas it is not consistent for the northern winter. This discrepancy would not disappear even in a dipole magnetosphere model because the flux tube convergence tends to yield a larger magnetic intensity in the hemisphere with larger main magnetic field intensity. We need to enhance the effect of the partial reflection of the Alfvén wave by the field-aligned increase in $V_{A}$ in order to ob- 
tain a result consistent with the observations. We need more realistic model of $V_{A}$ along a field line in addition to using a dipole model. As well, the validity of the thin-ionosphere assumption should be re-considered when the lower-latitude phenomena are treated.

\section{Discussion and Conclusion}

\subsection{Effects of the height of the ionosphere and the lati- tudinal wave number}

Equation (13) indicates that the height of the ionosphere (d) and the latitudinal wave number $\left(k_{x}\right)$ also affect the ground magnetic perturbation. We can estimate quantitatively how variations of $d$ and $k_{x}$ affect the ionospheric electric field; we shall consider the static and inductive cases because this classification is important for assessing the north-south asymmetry of the conjugate ground magnetic perturbations.

In the present study, we use $d$ (the height of the ionosphere normalized to the half-length of the field line) $=0.01$. When the actual height of the ionosphere is assumed to be about $100 \mathrm{~km}$, the length of the magnetic field line is $20000 \mathrm{~km}$ corresponding to $L \simeq 3$. At higher latitudes $(L>3), d$ should be smaller than 0.01. Equations (13) or (14) indicates that smaller $d$ (i.e., the case of the higher latitude) reduces the inductive electric field. Therefore, we can conclude that the magnetic shielding effect due to the ionospheric Hall current tends to appear in the more enhanced Hall conductivity. From Eq. (14), we also find that smaller $d$ enhances the ground magnetic perturbation. In evaluating latitudinal variations in the conjugate asymmetry of the ground magnetic perturbations in the latter part of this section, $d$ is adjusted to the field line length at the relevant latitude.

Next, we briefly consider the latitudinal width of standing Alfvén oscillations. Strictly speaking, the pure standing Alfvén oscillation does not have the latitudinal width. However, such an oscillation has no magnetic effect on the ground because of severe spatial attenuation in the neutral atmosphere (Hughes and Southwood, 1976a). Only the Alfvén wave coupled with the fast magnetosonic mode wave has finite latitudinal width (Southwood, 1974). However, it is still quite difficult to treat self-consistently the interaction between the coupled oscillation and the anisotropically conducting ionosphere. Bearing in mind that the behavior of the oscillation at the resonant field line is almost Alfvénic, we considered the ionosphere-Alfvén wave interaction as a working model for ground-based observations. Therefore, the latitudinal width is assigned here as a parameter. Similar to the case for $d$, it is possible to investigate the effect of the latitudinal width $\left(\simeq 1 / k_{x}\right)$ based on Eqs. (13)-(16). As for the disturbances with the latitudinal extent larger than $d$, we can see that $k_{x}$ almost disappears for $k_{x} d \ll 1$. This fact indicates that the variation of $k_{x}$ ( $\simeq 1 /$ latitudinal width) has no significant influence on the ionospheric electric field.

\subsection{Conclusion}

We investigated the ground magnetic perturbation associated with standing Alfvén oscillations using a model magnetosphere with the anisotropically conducting ionosphere. In this model, the main magnetic field lines are straight and have an arbitrary inclination angle to the ionosphere. First, the general features of the ground magnetic perturbation associated with standing Alfvén oscillations were investi- gated in the static condition of the ionospheric conductivity $\left(\Sigma_{P}>\Sigma_{H}\right)$ and in the inductive case $\left(\Sigma_{P}<\Sigma_{H}\right)$. Effects of the ionospheric conductivity, inclination angle and the equatorial plane-ionosphere $V_{A}$ ratio are also investigated. The main results are summarized as follows.

1) In the static condition, the intensity of the ground magnetic perturbation normalized to the magnetic perturbation just above the ionosphere is proportional to $\Sigma_{H} / \Sigma_{P}$. In the inductive condition, it is proportional to $1 / \Sigma_{H}$. When $\Sigma_{H}$ in the both ionospheres are the same, the ground magnetic perturbation is larger in the hemisphere with smaller $\Sigma_{P}$. When $\Sigma_{P}$ in the both ionospheres are the same, it is larger in the hemisphere with larger $\Sigma_{H}$ in the static condition and smaller in the hemisphere with smaller $\Sigma_{H}$ in the inductive condition.

2) The magnetic inclination controls the ground magnetic perturbation normalized to the magnetic perturbation just above the ionosphere mainly in the static condition. If the ionospheric conductivities of both hemispheres are same, the ground magnetic perturbation is larger in the hemisphere with smaller inclination. In the inductive condition, on the other hand, the ground magnetic perturbations in the both hemispheres are same even if the inclination angles are different.

3) The intensity of the ground magnetic perturbation normalized to the magnetic perturbation just above the ionosphere decreases with the Alfvén speed in the ionosphere mainly in the inductive condition. The ground magnetic perturbation is larger in the hemisphere with the smaller Alfvén speed in the ionosphere due to the partial reflection of the Alfvén wave within the trapezoid model that does not have curved magnetic field lines. This partial reflection effect is counteracted by the magnetic flux convergence associated with the dipole magnetic field, although this effect is excluded in the present model. As a result, the asymmetry of the ground magnetic perturbation is independent of the ionospheric condition (static or inductive). Note that the north-south asymmetry of the ground magnetic perturbations derived from the partial reflection mechanism may involve seasonal variation because $V_{A}$ is affected not only by the main magnetic field intensity but also by the plasma density that exhibits seasonal variation. On the other hand, the magnetic flux tube convergence effect produces a DC bias in the asymmetry independent of season.

In the next part, we evaluate asymmetry of the conjugate ground magnetic perturbations by using the ionospheric conductivity based on the IRI model as well as the fieldaligned $V_{A}$ profile and magnetic inclination based on the IGRF model. The magnetic flux convergence effect that is excluded in the present trapezoid model is qualitatively discussed. The results are as follows.

1) The calculation based on realistic parameters reveals that the fundamental mode has a static electric field in the ionosphere. Therefore, the two-step procedure of obtaining the ground magnetic perturbation from standing Alfvén oscillations (Allan, 1995) is valid for the fun- 
damental mode. On the other hand, the higher harmonic modes represent the inductive case. The Hall conductivity plays an essential role in the ionospheric reflection and transmission of standing Alfvén oscillations.

2) The north-south asymmetry of the ground magnetic perturbations depends on not only the $L$-value but also the magnetic longitude; this is because the ionosphere and magnetic field conditions are not uniform in the longitude.

The model used here is still quite artificial. The present trapezoid model ignores the curvature of the main magnetic field lines. When the curvature is taken into account, a decrease in the cross section of the magnetic flux tube toward the ionosphere may amplify the electromagnetic field perturbation associated with the Alfvén wave propagating toward the ionosphere. We need to employ the dipole magnetosphere model in the next study to consider quantitatively these effects.

Only the stationary standing Alfvén oscillation is considered in the present paper. Note that this is also quite idealized picture of the actual phenomena in which we need to take into account the other physical mechanisms (for example, the phase mixing effect) when we try to compare the observed data and the present numerical results. However, it seems interesting to compare the numerical results with observations by evaluating the magnetospheric magnetic perturbation from the ground based magnetic perturbation. The ground observations are now continuously carried out using a network of stations. Combination of the ground-based observations and the quantitative numerical model should open a new stage of research in this field.

Acknowledgments. One of authors (H.N) are very grateful for encouragement by Prof. T. Araki of Kyoto University. He is also deeply indebted to Prof. G. Rostoker of Nagoya University for his fruitful discussion and reviewing this manuscript. Thanks are also due to Dr. M. Takeda of WDC-C2 for geomagnetism in Kyoto University for his help of calculating the ionospheric conductivity. Numerical calculations were performed by the Computer Center of the National Institute of Polar Research. This work was supported by the Grant-in-Aid for Scientific Research (c), No. 09640532, from The Ministry of Education, Science and Culture.

\section{References}

Allan, W., Transient ULF pulsations: time dependence of magnetic fields observed at the ground, Ann. Geophys., 13, 938-945, 1995.

Allan, W. and F. B. Knox, A dipole field model for axisymmetric Alfvén waves with finite ionosphere conductivities, Planet. Space Sci., 27, 7985, 1979a.

Allan, W. and F. B. Knox, The effect of finite ionosphere conductivities on axisymmetric toroidal Alfvén wave resonances, Planet. Space Sci., 27, 939-950, 1979b.

Bilitza, D., International Reference Ionosphere 1990, National Space Science Data center/ World Data Center-A for Rockets and Satellites, 1990.

Buchert, S. C. and F. Budnik, Field-aligned current distributions generated by a divergent Hall current, Geophys. Res. Lett., 24, 297-300, 1997.

Chen, L. and A. Hasegawa, A theory of long-period magnetic pulsations 1. Steady state excitation of field line resonance, J. Geophys. Res., 79, 1024-1032, 1974

Fujita, S., Modification of magnetic signals of short-period pulsations by the ionosphere, Mem. Nat. Inst. Polar Res., Special Issue, 36, 287-296, 1985.

Hughes, W. J. and D. J. Southwood, The screening of micropulsation signals by the atmosphere and ionosphere, J. Geophys. Res., 81, 3234-3240, 1976a.
Hughes, W. J. and D. J. Southwood, An illustration of modification of geomagnetic pulsation structure by the ionosphere, J. Geophys. Res., 81, 3241-3247, 1976b.

IAGA Division V, Working Group 8, International Geomagnetic Field, 1995 Revision, J. Geomag. Geoelectr., 47, 1257-1261, 1995.

Inoue, Y. and S. Horowitz, Magneto-ionic coupling in an inhomogeneous anisotropic medium, Radio Sci., 1, 427-440, 1966a.

Inoue, Y. and S. Horowitz, Numerical solution of full-wave equation with mode coupling, Radio Sci., 1, 957-970, 1966b.

Itonaga, M., A. Yoshikawa, and T.-I. Kitamura, Interaction between hydromagnetic waves and the anisotropically conducting ionosphere, J. Geomag. Geoelectr., 47, 459-474, 1995.

Lee, D.-H. and R. L. Lysak, Magnetospheric ULF wave coupling in the dipole model: the impulsive excitation, J. Geophys. Res., 94, $17097-$ 17103, 1989.

Newton, R. S., D. J. Southwood, and W. J. Hughes, Damping of geomagnetic pulsations by the ionosphere, Planet. Space Sci., 26, 201-209, 1978.

Nishida, A., Ionospheric screening effect and storm sudden commencement, J. Geophys. Res., 69, 1861-1874, 1964.

Nishida, A., Geomagnetic Diagnosis of the Magnetosphere, 256 pp., Springer Verlag, New York, 1978.

Poulter, E. M. and W. Allan, Transient ULF pulsation decay rates observed by ground based magnetometers: The contribution of spatial integration, Planet. Space Sci., 33, 607-616, 1985.

Rees, D., COSPER International Reference Atmosphere: 1986 Part I: Thermosphere Models, Advances in Space Research, 8, 1988.

Saito, H., N. Sato, Y. Tonegawa, T. Yoshino, and T. Saemundsson, Seasonal and diurnal dependence of Pc 3-5 magnetic pulsation power at geomagnetically conjugate stations in the auroral zones, J. Geophys. Res., 94, 6945-6948, 1989.

Samson, J. C. and G. Rostoker, Latitude-dependent characteristics of highlatitude Pc 4 and Pc 5 micropulsations, J. Geophys. Res., 77, 6133-6144, 1972.

Southwood, D. J., Some features of field line resonances in the magnetosphere, Planet. Space Sci., 22, 483-491, 1974.

Tamao, T., The structure of three-dimensional hydromagnetic waves in a uniform cold plasma, J. Geomag. Geoelectr., 16, 89-114, 1964.

Tamao, T., Transmission and coupling resonance of hydromagnetic disturbances in the non-uniform earth's magnetosphere, Sci. Rep. Res. Inst Tohoku Univ. Geophy., 17, 43-72, 1965.

Tamao, T., Magnetosphere-ionosphere interaction through hydromagnetic waves, Proc. Conf. Achievements of the IMS, 26-28 June 1984, Graz, Austria, ESA SP-217, 427-435, 1984.

Tamao, T., Direct contribution of oblique field-aligned currents to ground magnetic fields, J. Geophys. Res., 91, 183-189, 1986.

Yoshikawa, A. and M. Itonaga, Reflection of shear Alfvén waves at the ionosphere and the divergent Hall current, Geophys. Res. Lett., 23, 101104, 1996.

Yoshikawa, A., M. Itonaga, and T. Kitamura, Effect of the ionospheric induction current on magnetohydrodynamic waves in the magnetosphere, Proc. NIPR Symp. Upper Atmos. Phys., 8, 49-59, 1995.

Yoshikawa, A., M. Itonaga, S. Fujita, H. Nakata, and K. Yumoto, Eigenmode analysis of field line oscillations interacting with the ionosphereatmosphere-solid earth electromagnetic coupled system, J. Geophys Res., 104, 28437-28457, 1999.

Yumoto, K., Y. Tanaka, T. Oguti, K. Shiokawa, Y. Yoshimura, A. Isono, B. J. Fraser, F. W. Menk, J. W. Lynn, M. Seto, and $210^{\circ}$ MM Magnetic Observation Group, Globally coordinated magnetic observations along $210^{\circ}$ magnetic meridian during STEP period: 1 . Preliminary results of low-latitude Pc3's, J. Geomag. Geoelectr, 44, 261-276, 1992.

Yumoto, K., A. Isono, K. Shiokawa, H. Matsuoka, Y. Tanaka, F. W. Menk, B. J. Fraser, and $210^{\circ}$ Magnetic Meridian Magnetic Observation Group, Global cavity mode-like and localized field-line Pc3-4 oscillations stimulated by interplanetary impulses $(\mathrm{Si} / \mathrm{Sc})$ : Initial results from the $210^{\circ}$ MM magnetic observations, in Solar Wind Sources of Magnetospheric Ultra-Low-Frequency Waves, Geophys. Monogr., vol. 81, edited by M. J. Engebretson, K. Takahashi, and M. Scholer, pp. 335-344, AGU, Washington, D.C., 1994.

Ziesolleck, C. W. S., B. J. Fraser, F. W. Menk, and P. W. McNabb, Spatial characteristics of low-latitude Pc3-4 geomagnetic pulsations, J. Geophys Res., 98, 197-207, 1993.

H. Nakata (e-mail: nakata@stelab.nagoya-u.ac.jp), S. Fujita, A. Yoshikawa, M. Itonaga and K. Yumoto 